\title{
Covid-19 and Excess Mortality in Medicare Beneficiaries
}

7 Cleveland, $\mathrm{OH}$

8

9 Clinic, Cleveland, $\mathrm{OH}$

10

11

12

13

14

15

16 * Corresponding Author:

(1)

Scott Greenwald ${ }^{11^{*}}$, Nassib G Chamoun ${ }^{1 \pi}$, Paul J Manberg ${ }^{1 \pi}$, Josh Gray ${ }^{11}$, David Clain ${ }^{1 \pi}$, Kamal Maheshwari $2,3 \pi$, Daniel I. Sessler

\footnotetext{
${ }^{1}$ Health Data Analytics Institute, Dedham MA
}

${ }^{2}$ Department of Outcomes Research, Anesthesiology Institute, Cleveland Clinic,

${ }^{3}$ Department of General Anesthesiology, Anesthesiology Institute, Cleveland

Email: Scott.Greenwald@hda-institute.com (SG)

II These authors contributed equally to this work. 
medRxiv preprint doi: https://doi.org/10.1101/2021.04.07.21254793; this version posted April 10, 2021. The copyright holder for this preprint (which was not certified by peer review) is the author/funder, who has granted medRxiv a license to display the preprint in perpetuity.

\section{Abstract}

18 We estimated excess mortality in Medicare recipients with probable and confirmed

19 Covid-19 infections in the general community and amongst residents of long-term care

20 (LTC) facilities. We considered 28,389,098 Medicare and dual-eligible recipients from

21 one year before February 29, 2020 through September 30, 2020, with mortality followed

22 through November $30^{\text {th }}$, 2020. Probable and confirmed Covid-19 diagnoses,

23 presumably mostly symptomatic, were determined from ICD-10 codes. We developed a

24 Risk Stratification Index (RSI) mortality model which was applied prospectively to

25 establish baseline mortality risk. Excess deaths attributable to Covid-19 were estimated

26 by comparing actual-to-expected deaths based on historical comparisons and in closely

27 matched cohorts with and without Covid-19. 677,100 (2.4\%) beneficiaries had

28 confirmed Covid-19 and 2,917,604 (10.3\%) had probable Covid-19. 472,329 confirmed

29 cases were community living and 204,771 were in LTC. Mortality following a probable or

30 confirmed diagnosis in the community increased from an expected incidence of about

$314 \%$ to actual incidence of $7.5 \%$. In long-term care facilities, the corresponding increase

32 was from $20.3 \%$ to $24.6 \%$. The absolute increase was therefore similar at $3-4 \%$ in the

33 community and in LTC residents. The percentage increase was far greater in the

34 community (89\%) than among patients in chronic care facilities $(21 \%)$ who had higher

35 baseline risk. The LTC population without probable or confirmed Covid-19 diagnoses

36 experienced 38,932 excess deaths (35\%) compared to historical estimates. Limitations

37 in access to Covid-19 testing and disease under-reporting in LTC patients probably

38 were important factors, although social isolation and disruption in usual care

39 presumably also contributed. Remarkably, there were 31,360 fewer deaths than 
medRxiv preprint doi: https://doi.org/10.1101/2021.04.07.21254793; this version posted April 10, 2021. The copyright holder for this preprint (which was not certified by peer review) is the author/funder, who has granted medRxiv a license to display the preprint in perpetuity. It is made available under a CC-BY-NC 4.0 International license.

40 expected in community dwellers without probable or confirmed Covid-19 diagnoses,

41 representing a $6 \%$ reduction. Disruptions to the healthcare system and avoided medical

42 care were thus apparently offset by other factors, representing overall benefit. The

43 Covid-19 pandemic had marked effects on mortality, but the effects were highly context-

44 dependent.

46 Introduction

47 The Covid-19 pandemic has profoundly influenced US healthcare, especially among

48 Medicare recipients who are mostly at least 65 years old. By March 1, 2021, SARS-

49 CoV-2, the virus responsible for Covid-19, had already infected more than 29 million

50 Americans and more than 500,000 died following infection.(1) However, many people

51 infected with Covid-19 are never tested or have false-negative test results;

52 consequently, the true toll of Covid-19 remains uncertain. Furthermore, while the clinical

53 course is sometimes apparent, Covid-19 also kills people by worsening chronic

54 conditions, with those deaths often being attributed to other causes. Especially early in

55 the pandemic, due to limited testing availability, it was difficult to differentiate deaths

56 caused by Covid-19 from those that may have occurred naturally due to underlying

57 health conditions. It is thus apparent that many people who died consequent to Covid-

5819 infections may not have been diagnosed with the condition or may have died due to 59 underlying causes. 
medRxiv preprint doi: https://doi.org/10.1101/2021.04.07.21254793; this version posted April 10, 2021. The copyright holder for this preprint (which was not certified by peer review) is the author/funder, who has granted medRxiv a license to display the preprint in perpetuity. It is made available under a CC-BY-NC 4.0 International license .

60 Several teams have estimated "excess" mortality due to Covid-19 by comparing weekly 61 observed death totals with those that occurred in a prior year. For example, Chen et al 62 estimated that from March 1 through August 22, 2020, 146,557 deaths were recorded in 63 California, with an estimated 19,806 (95\% Cl: 16,364, 23,210) deaths in excess of those 64 predicted by historical trends.(2) Similarly, Faust et al estimated that from March 1, 652020 , to July 31,2020 , a total of 76,088 all-cause deaths occurred among US adults 66 aged 25 to 44 years, which was 11,899 more than the expected 64,189 deaths based 67 on a previous year (incident rate ratio, $1.19[95 \% \mathrm{Cl}, 1.14-1.23]) .(3)$ Rossen et al 68 estimated excess mortality from January 26 through October 3 to have decreased $2 \%$ 69 for the youngest subjects (aged $<25$ years) but increased $14.4 \%$ for those $45-64$ years, $7021.1 \%$ for those $65-74$ years, $21.5 \%$ for those $75-84$ years, and $14.7 \%$ in subjects $71 \geq 85$ years old.(4) These reports suggest that all-cause mortality in the first six months of

72 the pandemic increased by about $15-20 \%$. . However, historical comparisons do not

73 account for risk at an individual level which may be useful to determine true excess

74 mortality.

75 On a broad population basis, many risk factors for Covid-19 are now well recognized.

76 For example, the CDC identifies eleven conditions that augment risk for severe forms of

77 Covid-19.(5) Chronic conditions such as cancer and dementia are reported to be among

78 the most important contributors.(6-8) It is clear that older members of the population are

79 at special risk, although to some extent age may be a surrogate for accumulated 80 comorbidities. However, it is difficult to extrapolate from population risk to individual risk 81 since many people exhibit various combinations of risk factors for Covid-19 mortality, 82 and individual risks attributable to each condition are not necessarily additive. A robust 
medRxiv preprint doi: https://doi.org/10.1101/2021.04.07.21254793; this version posted April 10, 2021. The copyright holder for this preprint (which was not certified by peer review) is the author/funder, who has granted medRxiv a license to display the preprint in perpetuity. It is made available under a CC-BY-NC 4.0 International license .

83 model that considers relevant individual conditions and predicts mortality risk from

84 Covid-19 infections would therefore be valuable.

85 Numerous groups have proposed individual risk models based on clinical outcomes in

86 various populations studied early in the pandemic, but a consensus model has yet to

87 emerge.(9-11) From a practical perspective, prediction models based on readily

88 available administrative data (e.g., ICD-10 codes) will be most useful since more

89 granular information extracted from clinical health records are neither universally

90 available nor easy to obtain. Our primary goal was therefore to estimate excess risk-

91 specific mortality in people with probable and confirmed Covid-19 infections.

92 An additional consequence of the Covid-19 pandemic has been public health

93 quarantines that have severely disrupted healthcare delivery. The virus may therefore

94 also have caused indirect mortality because patients with chronic diseases and acute

95 exacerbations avoided seekin care due to fear of infection or because health services

96 were overwhelmed or otherwise limiting access. Furthermore, stress related to isolation

97 could increase medical morbidity and provoke suicide and substance abuse. In contrast,

98 some causes of death such as accidents and homicides may have diminished. The

99 extent to which delayed and disrupted healthcare for non-Covid-related conditions,

100 along with pandemic-related behavioral changes, contribute to mortality remains

101 unclear. Our secondary goal was therefore to estimate whether changes in mortality

102 occurred in people without probable or confirmed Covid-19 infections.

103 Because Covid-19 is especially lethal in older people, we considered stratification by 104 various age ranges for both our primary and secondary analyses. We also separately 
medRxiv preprint doi: https://doi.org/10.1101/2021.04.07.21254793; this version posted April 10, 2021. The copyright holder for this preprint (which was not certified by peer review) is the author/funder, who has granted medRxiv a license to display the preprint in perpetuity. It is made available under a CC-BY-NC 4.0 International license .

105 considered people residing in the community from those in long term care facilities, who

106 are expected to have a higher baseline mortality risk and thus may be especially 107 susceptible to Covid-19 infections.

\section{Methods}

109 Data analysis was conducted on the Center for Medicare and Medicaid Services (CMS)

110 Research Identifiable File (RIF) data using SAS Enterprise Guide (Version 7.15) under

111 a special Data Use Agreement (DUA). This project was determined to be exempt from

112 informed consent requirements by the New England Institutional Review Board. Final

113 data analysis of the full cohort was conducted from January 10 to March 11,2021 . This

114 study followed the Strengthening the Reporting of Observational Studies in

115 Epidemiology (STROBE) reporting guideline for cohort studies.(12)

116 Individual subject data used for our analysis are available to certain stakeholders as

117 allowed by federal regulations and CMS policy. Requests for access to data to replicate

118 these findings require an approved research protocol and DUA with CMS. For more

119 information, contact the Research Data Assistance Center (ResDAC,

120 http://www.resdac.org).

121 Mortality predictions and outcomes were referenced to an anchor date of Feb 29, 2020,

122 just before the initial wave of documented Covid-19 cases and the week when the first

123 case of potential community spread Covid-19 was reported by CDC. We recognize that

124 undiagnosed cases may have occurred previously, but it is unlikely that there were 125 many. 
medRxiv preprint doi: https://doi.org/10.1101/2021.04.07.21254793; this version posted April 10, 2021. The copyright holder for this preprint (which was not certified by peer review) is the author/funder, who has granted medRxiv a license to display the preprint in perpetuity. It is made available under a CC-BY-NC 4.0 International license .

126 We used full Medicare fee-for-service and dual eligible (Medicaid and Medicare) files

127 one year before the anchor date through September 30, 2020, with mortality outcomes

128 reported through November $30^{\text {th }}, 2020$ for the primary analysis of Covid-19 outcomes.

129 We identified beneficiaries with confirmed Covid-19 diagnoses consistent with CMS

130 guidance using ICD-10-CM codes for Covid-19 (B97.29 before April 1, 2020 and U07.1

131 thereafter) as a primary or secondary diagnosis between March 1, 2020 and September

132 30, 2020.(13) Probable Covid-19 infection cases were identified using ICD-10-CM

133 codes consistent with the CDC guidance (Z20.828) and WHO recommendations

134 (U07.2).(14, 15) Presumably most subjects with Covid-19 diagnoses were symptomatic,

135 although some may have been tested because of risk or exposure.

136 Demographic characteristics (age, sex, ethnicity, location of care, zip code derived 137 measures) and Medicare coverage information (dates of coverage, enrollment history)

138 were extracted for each subject. Beneficiaries were classified as belonging to the long139 term care/skilled nursing facility (LTC/SNF) cohort if their claims history indicated that

140 they had received services in a LTC/SNF setting at any time in February 2020. The

141 remaining beneficiaries were designated as community dwelling.

142 We included all Medicaid and Medicare participants alive as of the study anchor date $143(\mathrm{~N}=65,310,173)$. We excluded beneficiaries who had: 1) ages outside 18-99 years

$144(214,767)$; 2) non-continuous coverage of Medicare Part A or B $(12,445,567)$ or any

145 Medicare Part C coverage $(23,633,391)$ in the year before the Feb 29, 2020 anchor 146 date; 3) missing data for any variable used in the analysis (509,932); and, 147 4) inconsistent data $(117,418)$. The resulting $28,389,098$ beneficiaries included 677,100 148 with confirmed and 2,917,604 with probable diagnosis of Covid-19 (S1 Fig). Mortality 
medRxiv preprint doi: https://doi.org/10.1101/2021.04.07.21254793; this version posted April 10, 2021. The copyright holder for this preprint (which was not certified by peer review) is the author/funder, who has granted medRxiv a license to display the preprint in perpetuity.

It is made available under a CC-BY-NC 4.0 International license .

149 was assumed to have occurred on the date-of-death listed in the CMS Common

150 Medicare Environment which is continuously updated from various sources including

151 the Social Security Administration.

152 Risk Stratification

153 We used an adaptation of the Risk Stratification Index (RSI) to predict nine-month

154 mortality using the prior year's administrative claims. A model was developed using the

155 full fee-for-service 2018 population for training, with prospective validation of

156 performance on the 2019 dataset as previously described.(16) More detailed

157 descriptions of the model and prospective testing performance results are provided in

158 S1 File and S2 Fig, respectively. The resulting RSI model was then prospectively

159 applied to all eligible Medicare or dual-eligible beneficiaries as of February 29, 2020 to

160 derive individual RSI scores as of that date - that is, before Covid-19 infections were

161 confirmed in the United States.

162 For comparative purposes, a second model was similarly developed to predict nine-

163 month mortality from the presence of 27 individual chronic conditions as defined by

164 CMS.(17) Specifically, logistic regression (stepwise selection using $p$-in of $10^{-3}$, p-out of

$16510^{-2}$ ) was used to select significant predictors from a pool of candidate features (i.e., 27

166 chronic conditions, age, sex and dual-enrollment status) to create a predictive model

167 using the same training and prospective testing populations described in the previous

168 paragraph. Performance using baseline RSI values or the individual chronic conditions

169 model as predictors of outcomes in 2020 were then tested prospectively (S3 Fig).

\section{Analysis and Selection of Study Cohorts}


medRxiv preprint doi: https://doi.org/10.1101/2021.04.07.21254793; this version posted April 10, 2021. The copyright holder for this preprint (which was not certified by peer review) is the author/funder, who has granted medRxiv a license to display the preprint in perpetuity. It is made available under a CC-BY-NC 4.0 International license .

171 We conducted a progression of complementary inquiries. To test our primary

172 hypothesis, we first identified the main study cohorts of beneficiaries with diagnoses of

173 probable or confirmed Covid-19, and then subdivided them based on location of service

174 (community or LTC/SNF) as of February 29, 2020. Within each cohort, we determined

175 9-month mortality between the anchor date and November 30, 2020.

176 Our goal was to first define associations between baseline demographic characteristics

177 and health status as characterized by RSI with the risk of mortality following a Covid-19

178 diagnosis in the overall at-risk population and in pre-defined subpopulations. We initially

179 compared differences in mean baseline RSI scores between survivors and non-

180 survivors, then used univariable and multivariable regression modeling to estimate the

181 relative importance of baseline demographic factors, chronic conditions, and RSI scores

182 as independent predictors of mortality. A similar analysis was conducted to identify risk

183 factors associated with a confirmed diagnosis of Covid-19. We also determined the

184 association between RSI and observed mortality by beneficiary age group and location.

\section{Estimation of Expected Mortality}

186 Two independent methods were used to estimate expected 2020 mortality in our study

187 population. A historical comparison allowed us to compare year-over-year changes in 188 mortality in Medicare recipients and thus characterize overall effects of Covid-19 and 189 quarantine-induced restrictions in healthcare access on mortality. A case-matched 190 analysis provided an alternate estimate of Covid-19-related excess mortality within the 1919 months of 2020 that we considered. 


\section{Historical Comparison}

195 As in previous studies, $(3,4)$ the first approach estimated expected 2020 mortality

196 figures from historical records. The daily observed mortality for the Medicare population

197 from 2017-2019 was used to prepare a model with optimal fit to capture seasonality and

198 account for annual trends using a three-year moving average adjustment (S2 Fig). This

199 approach better estimates expected mortality than relying on a single year-over-year

200 comparison because the model better captures year-to-year fluctuations consequent to

201 severity of yearly influenza outbreaks and other factors. We calculated predicted

202 mortality for each individual and designated the sum as the historically expected

203 mortality in each subpopulation. Excess deaths thus equaled the difference between

204 observed 2020 deaths and the historical projection of expected deaths (actual minus 205 expected).

\section{Case Matching, Digital Twins}

207 A second method used case matching or "digital twinning" to estimate excess mortality 208 in exposed subjects compared to concurrent controls who had closely matched health 209 profiles. Beneficiaries receiving a diagnosis of probable or confirmed Covid-19 were

210 pairwise exactly matched 1:1 on Feb 29, 2020 with beneficiaries without a Covid-19

211 diagnosis based on sex, age (within 1-year), ethnicity, location of services in Feb 2020

212 (community or LTC/SNF), along with RSI as a propensity matching factor (within $0.1 \%$ ). 
medRxiv preprint doi: https://doi.org/10.1101/2021.04.07.21254793; this version posted April 10, 2021. The copyright holder for this preprint (which was not certified by peer review) is the author/funder, who has granted medRxiv a license to display the preprint in perpetuity.

It is made available under a CC-BY-NC 4.0 International license .

213 Because the eligible Medicare population is large, we successfully matched almost the

214 entire infected population. Excess deaths were estimated as the difference between the

215 observed number of deaths in probable or confirmed Covid-19 subjects and their

216 matched non-Covid-19 digital twins over the concurrent period. Matching may be more

217 reliable than the historical comparison for estimating true excess mortality because it

218 better accounts for population variation over time and accounts for the impact of

219 substantial disruptions in public health and everyday life caused by the pandemic

220 restrictions in 2020.

\section{Statistical Analysis}

222 We used SAS Enterprise Guide (Version 7.15) to build models and conduct analyzes.

223 We excluded patients who lacked any variable used in our analyses including birth date,

224 sex, race, Medicaid enrollment status, zip code, and baseline RSI. Additionally, we

225 excluded subjects whose records had inconsistent values among source files containing

226 similar variables such as birth date and sex.

227 Patient demographic and clinical characteristics are summarized descriptively. Baseline

228 characteristics were compared using $t$ or $x^{2}$ tests, as appropriate. Mortality rates within

229 the study period post-Covid-19 diagnosis are reported as odds ratios with $95 \%$

230 confidence intervals. $P$ values $<0.05$ defined statistical significance for both the primary

231 and secondary outcomes. No adjustments were made for multiple comparisons. Sample

232 size requirements were not estimated a priori because the intention was to include all

233 qualifying 2020 beneficiaries available in the $100 \%$ nationwide Medicare files. 


\section{Results}

\section{Patient characteristics and outcomes}

237 As of Feb 29, 2020, a total of 28,389,098 Medicare or dual eligible beneficiaries met 238 inclusion criteria for this study. Among them, 677,100 (2.4\%) beneficiaries had a 239 diagnosis of confirmed Covid-19 and 2,917,604 (10.3\%) had a diagnosis of probable

240 Covid-19 during the study period (S1 Fig). Among the confirmed cases, 472,329 were

241 in the Community group while 204,771 received care in a long-term care setting.

243 Table1 and Table 2 compare demographic and clinical profiles for various subgroups.

244 Compared to survivors, patients who died after a Covid-19 diagnosis were older, more 245 often male, not white, received Medicaid, lived in zip-codes associated with lower

246 median income, received services in February 2020 in a long-term care facility, and had 247 higher baseline risk of mortality as defined by RSI. Age and baseline RSI scores were 248 both strongly related to risk of infection and adverse Covid-19 outcomes. Residence in a 249 LTC/SNF location and presence of end-stage renal disease were strong risk factors for 250 acquiring a confirmed diagnosis of Covid-19 (S4 Fig). As shown in S5 Fig, RSI scores 251 were associated with increasing mortality in a consistent rank ordered manner across 252 each age group, thereby suggesting that RSI provides a significant and sensitive 253 measure of co-morbidities and mortality risk that is independent of age. 
255 Table 1. Comparison of baseline demographic characteristics of all study populations.

\begin{tabular}{|c|c|c|c|c|c|c|c|c|c|}
\hline & \multicolumn{9}{|c|}{ Group Size (N,\%) } \\
\hline Characteristic & $\begin{array}{c}\text { All } \\
\text { Beneficiaries } \\
(28,389,098)\end{array}$ & $\begin{array}{c}\text { COVID } \\
\text { Confirmed } \\
(677,100)\end{array}$ & $\operatorname{Pr}<Z$ & $\begin{array}{c}\text { Died } \\
(131,460)\end{array}$ & $\begin{array}{l}\text { Survived } \\
(545,640)\end{array}$ & $\begin{array}{c}\text { COVID } \\
\text { Probable } \\
(2,917,604)\end{array}$ & $\operatorname{Pr}<Z$ & $\begin{array}{c}\text { Died } \\
(214,602)\end{array}$ & $\begin{array}{c}\text { Survived } \\
(2,703,002)\end{array}$ \\
\hline \multicolumn{10}{|l|}{ Age Group } \\
\hline $18-55$ & $\begin{array}{c}1,938,606 \\
(6.8)\end{array}$ & $\begin{array}{c}43,637 \\
(6.4)\end{array}$ & $<.0001$ & $\begin{array}{c}3,070 \\
(2.3)\end{array}$ & $\begin{array}{c}40,567 \\
(7.4)\end{array}$ & $\begin{array}{c}210,430 \\
(7.2)\end{array}$ & $<.0001$ & $\begin{array}{c}6,155 \\
(2.9)\end{array}$ & $\begin{array}{c}204,275 \\
(7.6)\end{array}$ \\
\hline $56-65$ & $\begin{array}{c}1,912,571 \\
(6.7)\end{array}$ & $\begin{array}{c}57,655 \\
(8.5)\end{array}$ & $<.0001$ & $\begin{array}{c}7,630 \\
(5.8)\end{array}$ & $\begin{array}{c}50,025 \\
(9.2)\end{array}$ & $\begin{array}{c}224,790 \\
(7.7)\end{array}$ & $<.0001$ & $\begin{array}{c}12,503 \\
(5.8)\end{array}$ & $\begin{array}{c}212,287 \\
(7.9)\end{array}$ \\
\hline $66-70$ & $\begin{array}{c}6,900,760 \\
(24.3)\end{array}$ & $\begin{array}{c}118,536 \\
(17.5)\end{array}$ & $<.0001$ & $\begin{array}{c}12,145 \\
(9.2)\end{array}$ & $\begin{array}{c}106,391 \\
(19.5)\end{array}$ & $\begin{array}{c}643,015 \\
(22.0)\end{array}$ & $<.0001$ & $\begin{array}{c}22,527 \\
(10.5)\end{array}$ & $\begin{array}{c}620,488 \\
(23.0)\end{array}$ \\
\hline $71-75$ & $\begin{array}{c}6,707,830 \\
(23.6)\end{array}$ & $\begin{array}{c}123,535 \\
(18.2)\end{array}$ & $<.0001$ & $\begin{array}{c}16,740 \\
(12.7)\end{array}$ & $\begin{array}{c}106,795 \\
(19.6)\end{array}$ & $\begin{array}{c}645,540 \\
(22.1)\end{array}$ & $<.0001$ & $\begin{array}{c}30,035 \\
(14.0)\end{array}$ & $\begin{array}{c}615,505 \\
(22.8)\end{array}$ \\
\hline $76-80$ & $\begin{array}{c}4,688,185 \\
(16.5)\end{array}$ & $\begin{array}{c}103,925 \\
(15.3)\end{array}$ & $<.0001$ & $\begin{array}{c}20,067 \\
(15.3)\end{array}$ & $\begin{array}{c}83,858 \\
(15.4)\end{array}$ & $\begin{array}{c}476,297 \\
(16.3)\end{array}$ & $<.0001$ & $\begin{array}{c}34,007 \\
(15.8)\end{array}$ & $\begin{array}{c}442,290 \\
(16.4)\end{array}$ \\
\hline $81-85$ & $\begin{array}{c}3,140,391 \\
(11.1)\end{array}$ & $\begin{array}{c}88,874 \\
(13.1)\end{array}$ & $<.0001$ & $\begin{array}{c}22,686 \\
(17.3)\end{array}$ & $\begin{array}{l}66,188 \\
(12.1)\end{array}$ & $\begin{array}{c}333,813 \\
(11.4)\end{array}$ & $<.0001$ & $\begin{array}{c}36,602 \\
(17.1)\end{array}$ & $\begin{array}{c}297,211 \\
(11.0)\end{array}$ \\
\hline $86-90$ & $\begin{array}{c}1,914,030 \\
(6.7)\end{array}$ & $\begin{array}{c}74,295 \\
(11.0)\end{array}$ & $<.0001$ & $\begin{array}{c}23,529 \\
(17.9)\end{array}$ & $\begin{array}{c}50,766 \\
(9.3)\end{array}$ & $\begin{array}{c}224,030 \\
(7.7)\end{array}$ & $<.0001$ & $\begin{array}{c}35,949 \\
(16.8)\end{array}$ & $\begin{array}{c}188,081 \\
(7.0)\end{array}$ \\
\hline $91-95$ & $\begin{array}{c}947,362 \\
(3.3)\end{array}$ & $\begin{array}{c}50,455 \\
(7.5)\end{array}$ & $<.0001$ & $\begin{array}{c}18,665 \\
(14.2)\end{array}$ & $\begin{array}{c}31,790 \\
(5.8)\end{array}$ & $\begin{array}{c}125,819 \\
(4.3)\end{array}$ & $<.0001$ & $\begin{array}{c}27,433 \\
(12.8)\end{array}$ & $\begin{array}{c}98,386 \\
(3.6)\end{array}$ \\
\hline $96-99$ & $\begin{array}{c}239,363 \\
(0.8)\end{array}$ & $\begin{array}{c}16,188 \\
(2.4)\end{array}$ & $<.0001$ & $\begin{array}{c}6,928 \\
(5.3)\end{array}$ & $\begin{array}{c}9,260 \\
(1.7)\end{array}$ & $\begin{array}{c}33,870 \\
(1.2)\end{array}$ & $<.0001$ & $\begin{array}{c}9,391 \\
(4.4)\end{array}$ & $\begin{array}{c}24,479 \\
(0.9)\end{array}$ \\
\hline \multicolumn{10}{|l|}{ Sex } \\
\hline Female & $\begin{array}{c}15,538,151 \\
(54.7)\end{array}$ & $\begin{array}{c}385,062 \\
(56.9) \\
\end{array}$ & $<.0001$ & $\begin{array}{c}68,726 \\
(52.3)\end{array}$ & $\begin{array}{c}316,336 \\
(58.0) \\
\end{array}$ & $\begin{array}{c}1,655,208 \\
(56.7)\end{array}$ & $<.0001$ & $\begin{array}{c}110,919 \\
(51.7) \\
\end{array}$ & $\begin{array}{c}1,544,289 \\
(57.1)\end{array}$ \\
\hline Male & $\begin{array}{c}12,850,947 \\
(45.3)\end{array}$ & $\begin{array}{c}292,038 \\
(43.1) \\
\end{array}$ & $<.0001$ & $\begin{array}{c}62,734 \\
(47.7)\end{array}$ & $\begin{array}{c}229,304 \\
(42.0)\end{array}$ & $\begin{array}{c}1,262,396 \\
(43.3)\end{array}$ & $<.0001$ & $\begin{array}{c}103,683 \\
(48.3)\end{array}$ & $\begin{array}{c}1,158,713 \\
(42.9)\end{array}$ \\
\hline \multicolumn{10}{|l|}{ Race/Ethnicity } \\
\hline $\begin{array}{l}\text { American } \\
\text { Indian/Alaskan } \\
\text { Native }\end{array}$ & $\begin{array}{c}161,358 \\
(0.6)\end{array}$ & $\begin{array}{c}5,194 \\
(0.8)\end{array}$ & $<.0001$ & $\begin{array}{l}1,171 \\
(0.9)\end{array}$ & $\begin{array}{c}4,023 \\
(0.7)\end{array}$ & $\begin{array}{c}17,312 \\
(0.6)\end{array}$ & $<.0001$ & $\begin{array}{c}1,380 \\
(0.6)\end{array}$ & $\begin{array}{c}15,932 \\
(0.6)\end{array}$ \\
\hline
\end{tabular}




\begin{tabular}{|c|c|c|c|c|c|c|c|c|c|}
\hline $\begin{array}{l}\text { Asian/Pacific } \\
\text { Islander }\end{array}$ & $\begin{array}{c}766,965 \\
(2.7)\end{array}$ & $\begin{array}{c}16,894 \\
(2.5)\end{array}$ & $<.0001$ & $\begin{array}{c}3,679 \\
(2.8)\end{array}$ & $\begin{array}{c}13,215 \\
(2.4)\end{array}$ & $\begin{array}{c}57,742 \\
(2.0)\end{array}$ & $<.0001$ & $\begin{array}{c}4,270 \\
(2.0)\end{array}$ & $\begin{array}{c}53,472 \\
(2.0)\end{array}$ \\
\hline Black & $\begin{array}{c}2,308,293 \\
(8.1)\end{array}$ & $\begin{array}{c}97,484 \\
(14.4) \\
\end{array}$ & $<.0001$ & $\begin{array}{c}21,394 \\
(16.3)\end{array}$ & $\begin{array}{c}76,090 \\
(13.9) \\
\end{array}$ & $\begin{array}{c}263,726 \\
(9.0) \\
\end{array}$ & $<.0001$ & $\begin{array}{c}20,962 \\
(9.8) \\
\end{array}$ & $\begin{array}{c}242,764 \\
(9.0) \\
\end{array}$ \\
\hline Hispanic & $\begin{array}{c}1,577,063 \\
(5.6)\end{array}$ & $\begin{array}{c}65,953 \\
(9.7) \\
\end{array}$ & $<.0001$ & $\begin{array}{c}12,760 \\
(9.7) \\
\end{array}$ & $\begin{array}{c}53,193 \\
(9.7) \\
\end{array}$ & $\begin{array}{c}151,585 \\
(5.2) \\
\end{array}$ & $<.0001$ & $\begin{array}{c}9,898 \\
(4.6) \\
\end{array}$ & $\begin{array}{c}141,687 \\
(5.2) \\
\end{array}$ \\
\hline $\begin{array}{l}\text { Non-Hispanic } \\
\text { White }\end{array}$ & $\begin{array}{l}22,806,998 \\
(80.3)\end{array}$ & $\begin{array}{c}477,687 \\
(70.5)\end{array}$ & $<.0001$ & $\begin{array}{c}90,660 \\
(69.0)\end{array}$ & $\begin{array}{c}387,027 \\
(70.9)\end{array}$ & $\begin{array}{l}2,352,811 \\
(80.6)\end{array}$ & $<.0001$ & $\begin{array}{c}175,365 \\
(81.7)\end{array}$ & $\begin{array}{l}2,177,446 \\
(80.6)\end{array}$ \\
\hline Other & $\begin{array}{c}228,402 \\
(0.8)\end{array}$ & $\begin{array}{c}5,164 \\
(0.8)\end{array}$ & 0.0001 & $\begin{array}{c}1,004 \\
(0.8)\end{array}$ & $\begin{array}{c}4,160 \\
(0.8)\end{array}$ & $\begin{array}{c}20,356 \\
(0.7)\end{array}$ & $<.0001$ & $\begin{array}{c}1,272 \\
(0.6)\end{array}$ & $\begin{array}{c}19,084 \\
(0.7)\end{array}$ \\
\hline Unknown & $\begin{array}{c}540,019 \\
(1.9)\end{array}$ & $\begin{array}{c}8,724 \\
(1.3)\end{array}$ & $<.0001$ & $\begin{array}{l}792 \\
(0.6)\end{array}$ & $\begin{array}{c}7,932 \\
(1.5)\end{array}$ & $\begin{array}{c}54,072 \\
(1.9)\end{array}$ & $<.0001$ & $\begin{array}{l}1,455 \\
(0.7)\end{array}$ & $\begin{array}{c}52,617 \\
(1.9)\end{array}$ \\
\hline \multicolumn{10}{|l|}{$\begin{array}{l}\text { Low } \\
\text { Income/Disabled } \\
\text { Status }\end{array}$} \\
\hline $\begin{array}{l}\text { Low Income or } \\
\text { Disabled }\end{array}$ & $\begin{array}{l}8,222,944 \\
(29.0)\end{array}$ & $\begin{array}{c}329,258 \\
(48.6)\end{array}$ & $<.0001$ & $\begin{array}{l}69,107 \\
(52.6)\end{array}$ & $\begin{array}{c}260,151 \\
(47.7)\end{array}$ & $\begin{array}{c}982,097 \\
(33.7)\end{array}$ & $<.0001$ & $\begin{array}{l}82,852 \\
(38.6)\end{array}$ & $\begin{array}{c}899,245 \\
(33.3)\end{array}$ \\
\hline $\begin{array}{l}\text { Not Low } \\
\text { Income/Disabled }\end{array}$ & $\begin{array}{c}20,166,154 \\
\quad(71.0)\end{array}$ & $\begin{array}{c}347,842 \\
(51.4)\end{array}$ & $<.0001$ & $\begin{array}{l}62,353 \\
(47.4)\end{array}$ & $\begin{array}{c}285,489 \\
(52.3)\end{array}$ & $\begin{array}{c}1,935,507 \\
(66.3)\end{array}$ & $<.0001$ & $\begin{array}{c}131,750 \\
(61.4)\end{array}$ & $\begin{array}{c}1,803,757 \\
(66.7)\end{array}$ \\
\hline \multicolumn{10}{|l|}{$\begin{array}{l}\text { Median } \\
\text { Household } \\
\text { Income } \\
\text { (Imputed from } \\
\text { Zip } \\
\text { code) } \\
\text { (Quintile) }\end{array}$} \\
\hline $20-40 \%$ & $\begin{array}{c}5,674,738 \\
(20.0)\end{array}$ & $\begin{array}{c}121,354 \\
(17.9)\end{array}$ & $<.0001$ & $\begin{array}{c}23,044 \\
(17.5)\end{array}$ & $\begin{array}{c}98,310 \\
(18.0)\end{array}$ & $\begin{array}{c}520,937 \\
(17.9)\end{array}$ & $<.0001$ & $\begin{array}{l}42,043 \\
(19.6)\end{array}$ & $\begin{array}{c}478,894 \\
(17.7)\end{array}$ \\
\hline $40-60 \%$ & $\begin{array}{c}5,679,860 \\
(20.0)\end{array}$ & $\begin{array}{c}122,059 \\
(18.0)\end{array}$ & $<.0001$ & $\begin{array}{c}23,585 \\
(17.9)\end{array}$ & $\begin{array}{c}98,474 \\
(18.0)\end{array}$ & $\begin{array}{c}533,097 \\
(18.3)\end{array}$ & $<.0001$ & $\begin{array}{c}41,889 \\
(19.5)\end{array}$ & $\begin{array}{c}491,208 \\
(18.2)\end{array}$ \\
\hline $60-80 \%$ & $\begin{array}{c}5,675,343 \\
(20.0)\end{array}$ & $\begin{array}{c}122,827 \\
(18.1)\end{array}$ & $<.0001$ & $\begin{array}{c}23,660 \\
(18.0)\end{array}$ & $\begin{array}{c}99,167 \\
(18.2)\end{array}$ & $\begin{array}{c}599,106 \\
(20.5)\end{array}$ & $<.0001$ & $\begin{array}{c}42,578 \\
(19.8)\end{array}$ & $\begin{array}{c}556,528 \\
(20.6)\end{array}$ \\
\hline Highest $20 \%$ & $\begin{array}{c}5,679,506 \\
(20.0)\end{array}$ & $\begin{array}{c}152,382 \\
(22.5)\end{array}$ & 01 & $\begin{array}{c}28,850 \\
(21.9)\end{array}$ & $\begin{array}{c}123,532 \\
(22.6)\end{array}$ & $\begin{array}{c}725,509 \\
(24.9)\end{array}$ & $<.0001$ & $\begin{array}{c}42,077 \\
(19.6)\end{array}$ & $\begin{array}{c}683,432 \\
(25.3)\end{array}$ \\
\hline Lowest 20\% & $\begin{array}{c}5,679,651 \\
(20.0)\end{array}$ & $\begin{array}{c}158,478 \\
(23.4)\end{array}$ & $<.0001$ & $\begin{array}{c}32,321 \\
(24.6)\end{array}$ & $\begin{array}{c}126,157 \\
(23.1)\end{array}$ & $\begin{array}{c}538,955 \\
(18.5)\end{array}$ & $<.0001$ & $\begin{array}{l}46,015 \\
(21.4)\end{array}$ & $\begin{array}{c}492,940 \\
(18.2)\end{array}$ \\
\hline
\end{tabular}




\begin{tabular}{|c|c|c|c|c|c|c|c|c|c|}
\hline $\begin{array}{l}\text { Community vs } \\
\text { LTC/SNF }\end{array}$ & & & & & & & & & \\
\hline Community & $\begin{array}{c}27,400,582 \\
(96.5)\end{array}$ & $\begin{array}{c}472,329 \\
(69.8)\end{array}$ & $<.0001$ & $\begin{array}{c}69,488 \\
(52.9) \\
\end{array}$ & $\begin{array}{c}402,841 \\
(73.8)\end{array}$ & $\begin{array}{c}2,676,502 \\
(91.7)\end{array}$ & $<.0001$ & $\begin{array}{c}166,800 \\
(77.7) \\
\end{array}$ & $\begin{array}{c}2,509,702 \\
(92.8)\end{array}$ \\
\hline $\begin{array}{l}\text { In Long term } \\
\text { care Facility or } \\
\text { SNF }\end{array}$ & $\begin{array}{c}988,516 \\
(3.5)\end{array}$ & $\begin{array}{c}204,771 \\
(30.2)\end{array}$ & $<.0001$ & $\begin{array}{c}61,972 \\
(47.1)\end{array}$ & $\begin{array}{c}142,799 \\
(26.2)\end{array}$ & $\begin{array}{c}241,102 \\
(8.3)\end{array}$ & $<.0001$ & $\begin{array}{c}47,802 \\
(22.3)\end{array}$ & $\begin{array}{c}193,300 \\
(7.2)\end{array}$ \\
\hline \multicolumn{10}{|l|}{ RSI (9mo) } \\
\hline $\begin{array}{l}\text { MEAN } \\
\text { (SD) }\end{array}$ & $\begin{array}{l}0.032 \\
(.071)\end{array}$ & $\begin{array}{l}0.096 \\
(.127)\end{array}$ & $<.0001$ & $\begin{array}{l}0.181 \\
(.153)\end{array}$ & $\begin{array}{l}0.075 \\
(.110)\end{array}$ & $\begin{array}{l}0.052 \\
(.095)\end{array}$ & $<.0001$ & $\begin{array}{l}0.168 \\
(.155)\end{array}$ & $\begin{array}{l}0.042 \\
(.082)\end{array}$ \\
\hline \multicolumn{10}{|l|}{$\begin{array}{l}\text { Mortality } \\
\text { (Through Nov } \\
\text { 30) }\end{array}$} \\
\hline Died & $\begin{array}{c}1,045,326 \\
(3.7)\end{array}$ & $\begin{array}{c}131,460 \\
(19.4)\end{array}$ & $<.0001$ & $\begin{array}{l}131,460 \\
(100.0)\end{array}$ & $\begin{array}{c}0 \\
(0.0)\end{array}$ & $\begin{array}{c}214,602 \\
(7.4)\end{array}$ & $<.0001$ & $\begin{array}{c}214,602 \\
(100.0)\end{array}$ & $\begin{array}{c}0 \\
(0.0)\end{array}$ \\
\hline Survived & $\begin{array}{c}27,343,772 \\
(96.3)\end{array}$ & $\begin{array}{c}545,640 \\
(80.6)\end{array}$ & $<.0001$ & $\begin{array}{c}0 \\
(0.0)\end{array}$ & $\begin{array}{c}545,640 \\
(100.0)\end{array}$ & $\begin{array}{c}2,703,002 \\
(92.6)\end{array}$ & $<.0001$ & $\begin{array}{c}0 \\
(0.0)\end{array}$ & $\begin{array}{c}2,703,002 \\
(100.0)\end{array}$ \\
\hline
\end{tabular}

${ }^{*} \mathrm{p}$-values represent the level of significance comparing attributes between Confirmed and Probable Covid infected

257 populations relative to all Beneficiaries.

258 Confirmed Covid-19 cases were identified consistent with CMS guidance using ICD-10-CM codes for Covid-19 (B97.29

259 before April 1, 2020 and U07.1 thereafter) as a primary or secondary diagnosis between March 1, 2020 and September

$26030,2020 .^{12}$ Probable Covid-19 infection cases were identified using ICD-10-CM codes consistent with the CDC guidance

261 (Z20.828) and WHO recommendations (U07.2). ${ }^{13,14}$ The baseline risk of 9-month mortality defined by the Risk

262 Stratification Index (RSI) calculated on February 29, 2020 was $3.2 \%$ in the entire population and significantly higher

263 among those who died compared to those who survived. 
Table 2. Comparison of baseline demographic characteristics for No Covid-19, Confirmed Covid-19 and Probable

265 Covid-19 populations in the Community and LTC/SNF subgroups.

\begin{tabular}{|c|c|c|c|c|c|c|c|c|}
\hline \multirow[b]{3}{*}{ Characteristic } & \multicolumn{8}{|c|}{$\begin{array}{l}\text { Study group, No. } \\
(\%)\end{array}$} \\
\hline & \multicolumn{4}{|c|}{ Community } & \multicolumn{4}{|c|}{ SNF/LTC } \\
\hline & $\begin{array}{l}\text { No COVID } \\
(24,251,751)\end{array}$ & $\begin{array}{c}\begin{array}{c}\text { COVID } \\
\text { Confirmed }\end{array} \\
(472,329)\end{array}$ & $\begin{array}{c}\text { COVID } \\
\text { Probable } \\
(2,676,502)\end{array}$ & $\begin{array}{c}\text { P. } \\
\text { value }^{*}\end{array}$ & $\begin{array}{l}\text { No COVID } \\
(542,643)\end{array}$ & $\begin{array}{c}\text { COVID } \\
\text { Confirmed } \\
(204,771)\end{array}$ & $\begin{array}{c}\text { COVID } \\
\text { Probable } \\
(241,102)\end{array}$ & $\begin{array}{c}\text { P- } \\
\text { value* }\end{array}$ \\
\hline \multicolumn{9}{|l|}{ Age Group } \\
\hline $18-55$ & $\begin{array}{c}1,663,926 \\
(6.9)\end{array}$ & $\begin{array}{c}36,472 \\
(7.7)\end{array}$ & $\begin{array}{c}200,110 \\
(7.5)\end{array}$ & \multirow{9}{*}{$<.0001$} & $\begin{array}{c}20,613 \\
(3.8)\end{array}$ & $\begin{array}{l}7,165 \\
(3.5)\end{array}$ & $\begin{array}{c}10,320 \\
(4.3)\end{array}$ & \multirow{9}{*}{$<.0001$} \\
\hline $56-65$ & $\begin{array}{l}1,595,016 \\
(6.6)\end{array}$ & $\begin{array}{c}40,231 \\
(8.5)\end{array}$ & $\begin{array}{c}205,372 \\
(7.7)\end{array}$ & & $\begin{array}{c}35,110 \\
(6.5)\end{array}$ & $\begin{array}{c}17,424 \\
(8.5)\end{array}$ & $\begin{array}{c}19,418 \\
(8.1)\end{array}$ & \\
\hline $66-70$ & $\begin{array}{c}6,096,885 \\
(25.1)\end{array}$ & $\begin{array}{l}98,565 \\
(20.9)\end{array}$ & $\begin{array}{c}621,310 \\
(23.2)\end{array}$ & & $\begin{array}{c}42,324 \\
(7.8)\end{array}$ & $\begin{array}{c}19,971 \\
(9.8)\end{array}$ & $\begin{array}{c}21,705 \\
(9.0)\end{array}$ & \\
\hline $71-75$ & $\begin{array}{c}5,881,587 \\
(24.3)\end{array}$ & $\begin{array}{l}99,089 \\
(21.0)\end{array}$ & $\begin{array}{c}618,427 \\
(23.1) \\
\end{array}$ & & $\begin{array}{l}57,168 \\
(10.5)\end{array}$ & $\begin{array}{c}24,446 \\
(11.9)\end{array}$ & $\begin{array}{l}27,113 \\
(11.2)\end{array}$ & \\
\hline $76-80$ & $\begin{array}{c}4,036,407 \\
(16.6)\end{array}$ & $\begin{array}{l}75,063 \\
(15.9)\end{array}$ & $\begin{array}{c}443,657 \\
(16.6)\end{array}$ & & $\begin{array}{l}71,556 \\
(13.2)\end{array}$ & $\begin{array}{c}28,862 \\
(14.1) \\
\end{array}$ & $\begin{array}{l}32,640 \\
(13.5)\end{array}$ & \\
\hline $81-85$ & $\begin{array}{l}2,626,817 \\
(10.8)\end{array}$ & $\begin{array}{l}55,687 \\
(11.8)\end{array}$ & $\begin{array}{c}294,796 \\
(11.0)\end{array}$ & & $\begin{array}{l}90,887 \\
(16.7)\end{array}$ & $\begin{array}{l}33,187 \\
(16.2)\end{array}$ & $\begin{array}{l}39,017 \\
(16.2)\end{array}$ & \\
\hline $86-90$ & $\begin{array}{l}1,510,656 \\
(6.2)\end{array}$ & $\begin{array}{c}38,824 \\
(8.2)\end{array}$ & $\begin{array}{c}180,497 \\
(6.7)\end{array}$ & & $\begin{array}{c}105,049 \\
(19.4)\end{array}$ & $\begin{array}{l}35,471 \\
(17.3)\end{array}$ & $\begin{array}{l}43,533 \\
(18.1)\end{array}$ & \\
\hline $91-95$ & $\begin{array}{c}683,500 \\
(2.8)\end{array}$ & $\begin{array}{c}22,331 \\
(4.7)\end{array}$ & $\begin{array}{c}90,337 \\
(3.4)\end{array}$ & & $\begin{array}{l}87,588 \\
(16.1)\end{array}$ & $\begin{array}{l}28,124 \\
(13.7)\end{array}$ & $\begin{array}{l}35,482 \\
(14.7)\end{array}$ & \\
\hline $96-99$ & $\begin{array}{c}156,957 \\
(0.6)\end{array}$ & $\begin{array}{l}6,067 \\
(1.3)\end{array}$ & $\begin{array}{c}21,996 \\
(0.8)\end{array}$ & & $\begin{array}{c}32,348 \\
(6.0)\end{array}$ & $\begin{array}{c}10,121 \\
(4.9) \\
\end{array}$ & $\begin{array}{c}11,874 \\
(4.9)\end{array}$ & \\
\hline \multicolumn{9}{|l|}{ Sex } \\
\hline Female & $\begin{array}{c}13,146,555 \\
(54.2)\end{array}$ & $\begin{array}{c}256,524 \\
(54.3)\end{array}$ & $\begin{array}{c}1,498,825 \\
(56.0)\end{array}$ & $<.0001$ & $\begin{array}{c}351,326 \\
(64.7)\end{array}$ & $\begin{array}{c}128,538 \\
(62.8)\end{array}$ & $\begin{array}{c}156,383 \\
(64.9)\end{array}$ & $<.0001$ \\
\hline
\end{tabular}




\begin{tabular}{|c|c|c|c|c|c|c|c|c|}
\hline Male & $\begin{array}{c}11,105,196 \\
(45.8)\end{array}$ & $\begin{array}{c}215,805 \\
(45.7)\end{array}$ & $\begin{array}{c}1,177,677 \\
(44.0)\end{array}$ & & $\begin{array}{c}191,317 \\
(35.3)\end{array}$ & $\begin{array}{c}76,233 \\
(37.2)\end{array}$ & $\begin{array}{l}84,719 \\
(35.1)\end{array}$ & \\
\hline \multicolumn{9}{|l|}{ Race/Ethnicity } \\
\hline $\begin{array}{l}\text { American } \\
\text { Indian/Alaskan } \\
\text { Native }\end{array}$ & $\begin{array}{c}136,028 \\
(0.6)\end{array}$ & $\begin{array}{c}4,440 \\
(0.9)\end{array}$ & $\begin{array}{c}16,237 \\
(0.6)\end{array}$ & \multirow{7}{*}{$<.0001$} & $\begin{array}{c}2,824 \\
(0.5)\end{array}$ & $\begin{array}{l}754 \\
(0.4)\end{array}$ & $\begin{array}{l}1,075 \\
(0.4)\end{array}$ & \multirow{7}{*}{$<.0001$} \\
\hline $\begin{array}{l}\text { Asian/Pacific } \\
\text { Islander }\end{array}$ & $\begin{array}{c}683,510 \\
(2.8)\end{array}$ & $\begin{array}{c}11,804 \\
(2.5)\end{array}$ & $\begin{array}{c}53,768 \\
(2.0)\end{array}$ & & $\begin{array}{c}8,819 \\
(1.6)\end{array}$ & $\begin{array}{l}5,090 \\
(2.5)\end{array}$ & $\begin{array}{c}3,974 \\
(1.6)\end{array}$ & \\
\hline Black & $\begin{array}{l}1,902,790 \\
(7.8)\end{array}$ & $\begin{array}{c}64,609 \\
(13.7)\end{array}$ & $\begin{array}{c}236,985 \\
(8.9)\end{array}$ & & $\begin{array}{c}44,293 \\
(8.2)\end{array}$ & $\begin{array}{c}32,875 \\
(16.1)\end{array}$ & $\begin{array}{l}26,741 \\
(11.1)\end{array}$ & \\
\hline Hispanic & $\begin{array}{c}1,337,729 \\
(5.5)\end{array}$ & $\begin{array}{c}50,565 \\
(10.7)\end{array}$ & $\begin{array}{c}140,218 \\
(5.2)\end{array}$ & & $\begin{array}{c}21,796 \\
(4.0)\end{array}$ & $\begin{array}{c}15,388 \\
(7.5)\end{array}$ & $\begin{array}{c}11,367 \\
(4.7)\end{array}$ & \\
\hline $\begin{array}{l}\text { Non-Hispanic } \\
\text { White }\end{array}$ & $\begin{array}{c}19,516,975 \\
(80.5)\end{array}$ & $\begin{array}{c}329,288 \\
(69.7)\end{array}$ & $\begin{array}{l}2,157,239 \\
(80.6)\end{array}$ & & $\begin{array}{c}459,525 \\
(84.7) \\
\end{array}$ & $\begin{array}{c}148,399 \\
(72.5)\end{array}$ & $\begin{array}{c}195,572 \\
(81.1) \\
\end{array}$ & \\
\hline Other & $\begin{array}{c}199,925 \\
(0.8)\end{array}$ & $\begin{array}{l}3,860 \\
(0.8)\end{array}$ & $\begin{array}{c}19,064 \\
(0.7)\end{array}$ & & $\begin{array}{c}2,957 \\
(0.5)\end{array}$ & $\begin{array}{l}1,304 \\
(0.6)\end{array}$ & $\begin{array}{l}1,292 \\
(0.5)\end{array}$ & \\
\hline Unknown & $\begin{array}{c}474,794 \\
(2.0)\end{array}$ & $\begin{array}{c}7,763 \\
(1.6)\end{array}$ & $\begin{array}{c}52,991 \\
(2.0)\end{array}$ & & $\begin{array}{c}2,429 \\
(0.4)\end{array}$ & $\begin{array}{l}961 \\
(0.5)\end{array}$ & $\begin{array}{l}1,081 \\
(0.4)\end{array}$ & \\
\hline \multicolumn{9}{|l|}{$\begin{array}{l}\text { Low } \\
\text { Income/Disabled } \\
\text { Status }\end{array}$} \\
\hline $\begin{array}{l}\text { Lowlncome or } \\
\text { Disabled }\end{array}$ & $\begin{array}{c}6,653,284 \\
(27.4)\end{array}$ & $\begin{array}{c}189,901 \\
(40.2)\end{array}$ & $\begin{array}{c}846,357 \\
(31.6)\end{array}$ & \multirow[b]{2}{*}{$<.0001$} & $\begin{array}{c}258,305 \\
(47.6)\end{array}$ & $\begin{array}{c}139,357 \\
(68.1)\end{array}$ & $\begin{array}{c}135,740 \\
(56.3)\end{array}$ & \multirow[b]{2}{*}{$<.0001$} \\
\hline $\begin{array}{l}\text { Not } \\
\text { Lowlncome/Disabled }\end{array}$ & $\begin{array}{l}17,598,467 \\
\quad(72.6)\end{array}$ & $\begin{array}{c}282,428 \\
(59.8)\end{array}$ & $\begin{array}{c}1,830,145 \\
(68.4)\end{array}$ & & $\begin{array}{c}284,338 \\
(52.4)\end{array}$ & $\begin{array}{c}65,414 \\
(31.9)\end{array}$ & $\begin{array}{c}105,362 \\
(43.7)\end{array}$ & \\
\hline \multicolumn{9}{|l|}{$\begin{array}{l}\text { Median Household } \\
\text { Income (Imputed } \\
\text { from } \\
\text { Zip code) (Quintile) }\end{array}$} \\
\hline $20-40 \%$ & $\begin{array}{l}4917968 \\
(20.3)\end{array}$ & $\begin{array}{l}86115 \\
(18.2)\end{array}$ & $\begin{array}{c}473870 \\
(17.7)\end{array}$ & \multirow{2}{*}{$<.0001$} & $\begin{array}{c}114479 \\
(21.1)\end{array}$ & $\begin{array}{l}35239 \\
(17.2)\end{array}$ & $\begin{array}{l}47067 \\
(19.5)\end{array}$ & \multirow{2}{*}{$<.0001$} \\
\hline $40-60 \%$ & $\begin{array}{c}4913071 \\
(20.3)\end{array}$ & $\begin{array}{c}84952 \\
(18.0)\end{array}$ & $\begin{array}{c}487217 \\
(18.2)\end{array}$ & & $\begin{array}{c}111633 \\
(20.6)\end{array}$ & $\begin{array}{c}37107 \\
(18.1)\end{array}$ & $\begin{array}{l}45880 \\
(19.0)\end{array}$ & \\
\hline
\end{tabular}




\begin{tabular}{|c|c|c|c|c|c|c|c|c|}
\hline $60-80 \%$ & $\begin{array}{c}4848516 \\
(20.0)\end{array}$ & $\begin{array}{c}85749 \\
(18.2)\end{array}$ & $\begin{array}{c}553075 \\
(20.7)\end{array}$ & & $\begin{array}{c}104894 \\
(19.3)\end{array}$ & $\begin{array}{c}37078 \\
(18.1)\end{array}$ & $\begin{array}{l}46031 \\
(19.1)\end{array}$ & \\
\hline Highest $20 \%$ & $\begin{array}{c}4701144 \\
(19.4)\end{array}$ & $\begin{array}{c}105045 \\
(22.2)\end{array}$ & $\begin{array}{c}675456 \\
(25.2)\end{array}$ & & $\begin{array}{c}100471 \\
(18.5)\end{array}$ & $\begin{array}{c}47337 \\
(23.1) \\
\end{array}$ & $\begin{array}{l}50053 \\
(20.8)\end{array}$ & \\
\hline Lowest 20\% & $\begin{array}{c}4871052 \\
(20.1)\end{array}$ & $\begin{array}{c}110468 \\
(23.4)\end{array}$ & $\begin{array}{c}486884 \\
(18.2)\end{array}$ & & $\begin{array}{c}111166 \\
(20.5)\end{array}$ & $\begin{array}{c}48010 \\
(23.4)\end{array}$ & $\begin{array}{c}52071 \\
(21.6)\end{array}$ & \\
\hline \multicolumn{9}{|l|}{$\begin{array}{l}\text { Community vs } \\
\text { LTC/SNF }\end{array}$} \\
\hline Community & $\begin{array}{c}24251751 \\
(100.0)\end{array}$ & $\begin{array}{c}472329 \\
(100.0) \\
\end{array}$ & $\begin{array}{c}2676502 \\
(100.0)\end{array}$ & \multirow[b]{2}{*}{$<.0001$} & $\begin{array}{c}0 \\
(0.0) \\
\end{array}$ & $\begin{array}{c}0 \\
(0.0) \\
\end{array}$ & $\begin{array}{c}0 \\
(0.0)\end{array}$ & \multirow[b]{2}{*}{$<.0001$} \\
\hline $\begin{array}{l}\text { In Long term care } \\
\text { Facility or SNF }\end{array}$ & $\begin{array}{c}0 \\
(0.0)\end{array}$ & $\begin{array}{c}0 \\
(0.0)\end{array}$ & $\begin{array}{c}0 \\
(0.0)\end{array}$ & & $\begin{array}{c}542643 \\
(100.0)\end{array}$ & $\begin{array}{c}204771 \\
(100.0)\end{array}$ & $\begin{array}{c}241102 \\
(100.0)\end{array}$ & \\
\hline \multicolumn{9}{|l|}{ RSI (9mo) } \\
\hline MEAN (SD) & $\begin{array}{l}0.024 \\
(.055) \\
\end{array}$ & $\begin{array}{l}0.048 \\
(.086)\end{array}$ & $\begin{array}{l}0.038 \\
(.077) \\
\end{array}$ & $<.0001$ & $\begin{array}{l}0.206 \\
(.154) \\
\end{array}$ & $\begin{array}{l}0.206 \\
(.137) \\
\end{array}$ & $\begin{array}{l}0.201 \\
(.141) \\
\end{array}$ & $<.0001$ \\
\hline \multicolumn{9}{|l|}{$\begin{array}{l}\text { Mortality } \\
\text { (Through Nov 30) }\end{array}$} \\
\hline Died & $\begin{array}{c}548556 \\
(2.3)\end{array}$ & $\begin{array}{c}69488 \\
(14.7)\end{array}$ & $\begin{array}{c}166800 \\
(6.2)\end{array}$ & \multirow{2}{*}{$<.0001$} & $\begin{array}{c}150708 \\
(27.8)\end{array}$ & $\begin{array}{c}61972 \\
(30.3)\end{array}$ & $\begin{array}{c}47802 \\
(19.8)\end{array}$ & \multirow{2}{*}{$<.0001$} \\
\hline Survived & $\begin{array}{c}23703195 \\
(97.7)\end{array}$ & $\begin{array}{c}402841 \\
(85.3)\end{array}$ & $\begin{array}{c}2509702 \\
(93.8)\end{array}$ & & $\begin{array}{c}391935 \\
(72.2)\end{array}$ & $\begin{array}{c}142799 \\
(69.7)\end{array}$ & $\begin{array}{c}193300 \\
(80.2)\end{array}$ & \\
\hline
\end{tabular}

${ }^{*} \mathrm{p}$-value: $<0.05$ indicates the distribution of patients among subgroups (or the mean values of metrics) of the No Covid-

267 19, Confirmed Covid-19 and Probable Covid-19 groups.

268 Subjects were categorized as "LTC/SNF" if they received services in either a Long-Term Care (LTC) or Skilled Nursing

269 Facility (SNF) in February 2020, otherwise they were categorized as receiving services in the "Community." Confirmed

270 Covid-19 cases were identified consistent with CMS guidance using ICD-10-CM codes for Covid-19 (B97.29 before April

271 1, 2020 and U07.1 thereafter) as a primary or secondary diagnosis between March 1, 2020 and September 30, $2020 .^{12}$ 
272 Probable Covid-19 infection cases were identified using ICD-10-CM codes consistent with the CDC guidance (Z20.828)

273 and WHO recommendations (U07.2). ${ }^{13,14}$ The baseline risk of 9-month mortality defined by the Risk Stratification Index

274 (RSI) calculated on February 29, 2020 was multiple times higher among subjects who received services in LTC/SNF than 275 in the community.

276 
medRxiv preprint doi: https://doi.org/10.1101/2021.04.07.21254793; this version posted April 10, 2021. The copyright holder for this preprint (which was not certified by peer review) is the author/funder, who has granted medRxiv a license to display the preprint in perpetuity.

278 Fig 1 presents the relative importance of factors that contributed to mortality in both 279 univariable and multivariable models. Quintiles of RSI, age, LTC/SNF services status, 280 sex, and race were the factors most associated with relative risk of mortality. Status of 281 lung cancer and end-stage renal disease appear to carry meaningful incremental risk 282 after adjustment. Mortality prediction models based primarily on baseline RSI levels 283 performed better than models based on the presence of individual chronic conditions for 284 predicting mortality risk (S3 Fig). Case matching identified a cohort of beneficiaries from 285 the general population who were closely matched with subjects who had a diagnosis of 286 probable or confirmed Covid-19 based on their RSI scores as of Feb 29, 2020 (Table $2873)$.

288 Fig 1. Forest plot showing the relative risk and $95 \% \mathrm{Cl}$ of significant predictors of 289 mortality of subjects with confirmed Covid-19. Confirmed Covid-19 cases were 290 identified consistent with CMS guidance using ICD-10-CM codes for Covid-19 (B97.29 291 before April 1, 2020 and U07.1 thereafter) as a primary or secondary diagnosis between 292 March 1, 2020 and September 30, 2020. ${ }^{12}$ Subjects were categorized as "LTC/SNF" if 293 they received services in either a Long Term Care (LTC) or Skilled Nursing Facility 294 (SNF) in February 2020, otherwise they were categorized as receiving services in the 295 "Community." Predictors were assessed at baseline (February 29, 2020) and include 296 quintiles of Risk Stratification Index (RSI), presence of chronic conditions, location of 297 services (LTC/SNF vs Community), and demographic variables (i.e., age, sex, race, and 298 quintiles of median household income imputed by zip code according to 2015 Census 299 data.) Variables not remaining in the adjusted model are indicated by the presence of 
300 empty parenthesis under the adjusted odds ratio. RSI, age, and location of services

301 were the strongest (unadjusted) predictors of mortality. RSI and age remain strong

302 predictors following adjustment; however, risks associated with having chronic

303 conditions were typically reduced when adjusted by the presence of RSI and other

304 factors. Status of Lung cancer and end-stage renal disease appear to carry meaningful

305 incremental risk after adjustment.

306 
Community and LTC/SNF subgroups.

\begin{tabular}{|c|c|c|c|c|c|c|c|c|}
\hline \multirow{4}{*}{ Characteristic } & \multicolumn{8}{|c|}{$\begin{array}{c}\text { Study group, No. } \\
(\%)\end{array}$} \\
\hline & \multicolumn{4}{|c|}{ Community } & \multicolumn{4}{|c|}{ LTC/SNF } \\
\hline & \multicolumn{2}{|c|}{ COVID Confirmed } & \multicolumn{2}{|c|}{ COVID Probable } & \multicolumn{2}{|c|}{ COVID Confirmed } & \multicolumn{2}{|c|}{ COVID Probable } \\
\hline & $\begin{array}{c}\text { Infected } \\
(470,622)\end{array}$ & $\begin{array}{c}\text { Control } \\
(470,622)\end{array}$ & $\begin{array}{c}\text { Infected } \\
(2,667,931)\end{array}$ & $\begin{array}{c}\text { Control } \\
(2,667,923)\end{array}$ & $\begin{array}{l}\text { Infected } \\
(186,671)\end{array}$ & $\begin{array}{l}\text { Control } \\
(186,671)\end{array}$ & $\begin{array}{l}\text { Infected } \\
(225,970)\end{array}$ & $\begin{array}{c}\text { Control } \\
(225,970)\end{array}$ \\
\hline \multicolumn{9}{|l|}{ Age Group } \\
\hline $18-55$ & $\begin{array}{c}35,622 \\
(7.6)\end{array}$ & $\begin{array}{c}35,622 \\
(7.6)\end{array}$ & $\begin{array}{c}195,609 \\
(7.3)\end{array}$ & $\begin{array}{c}195,609 \\
(7.3)\end{array}$ & $\begin{array}{c}4,591 \\
(2.5)\end{array}$ & $\begin{array}{c}4,591 \\
(2.5)\end{array}$ & $\begin{array}{c}6,669 \\
(3.0)\end{array}$ & $\begin{array}{c}6,669 \\
(3.0)\end{array}$ \\
\hline $56-65$ & $\begin{array}{c}40,029 \\
(8.5)\end{array}$ & $\begin{array}{c}40,029 \\
(8.5)\end{array}$ & $\begin{array}{c}204,327 \\
(7.7)\end{array}$ & $\begin{array}{c}204,327 \\
(7.7)\end{array}$ & $\begin{array}{c}14,739 \\
(7.9)\end{array}$ & $\begin{array}{c}14,739 \\
(7.9)\end{array}$ & $\begin{array}{c}16,995 \\
(7.5)\end{array}$ & $\begin{array}{c}16,995 \\
(7.5)\end{array}$ \\
\hline $66-70$ & $\begin{array}{c}98,466 \\
(20.9)\end{array}$ & $\begin{array}{c}98,466 \\
(20.9)\end{array}$ & $\begin{array}{c}620,578 \\
(23.3)\end{array}$ & $\begin{array}{c}620,576 \\
(23.3)\end{array}$ & $\begin{array}{c}17,537 \\
(9.4)\end{array}$ & $\begin{array}{c}17,537 \\
(9.4)\end{array}$ & $\begin{array}{c}19,728 \\
(8.7)\end{array}$ & $\begin{array}{c}19,728 \\
(8.7)\end{array}$ \\
\hline $71-75$ & $\begin{array}{c}98,994 \\
(21.0)\end{array}$ & $\begin{array}{c}98,994 \\
(21.0)\end{array}$ & $\begin{array}{c}617,788 \\
(23.2)\end{array}$ & $\begin{array}{c}617,784 \\
(23.2)\end{array}$ & $\begin{array}{c}21,884 \\
(11.7)\end{array}$ & $\begin{array}{c}21,884 \\
(11.7)\end{array}$ & $\begin{array}{c}25,150 \\
(11.1)\end{array}$ & $\begin{array}{c}25,150 \\
(11.1)\end{array}$ \\
\hline $76-80$ & $\begin{array}{l}74,957 \\
(15.9)\end{array}$ & $\begin{array}{l}74,957 \\
(15.9)\end{array}$ & $\begin{array}{c}443,190 \\
(16.6) \\
\end{array}$ & $\begin{array}{c}443,188 \\
(16.6)\end{array}$ & $\begin{array}{l}26,201 \\
(14.0)\end{array}$ & $\begin{array}{l}26,201 \\
(14.0)\end{array}$ & $\begin{array}{c}30,893 \\
(13.7)\end{array}$ & $\begin{array}{c}30,893 \\
(13.7)\end{array}$ \\
\hline $81-85$ & $\begin{array}{c}55,589 \\
(11.8)\end{array}$ & $\begin{array}{c}55,589 \\
(11.8)\end{array}$ & $\begin{array}{c}294,423 \\
(11.0)\end{array}$ & $\begin{array}{c}294,423 \\
(11.0)\end{array}$ & $\begin{array}{c}31,053 \\
(16.6)\end{array}$ & $\begin{array}{c}31,053 \\
(16.6)\end{array}$ & $\begin{array}{c}37,647 \\
(16.7)\end{array}$ & $\begin{array}{c}37,647 \\
(16.7)\end{array}$ \\
\hline $86-90$ & $\begin{array}{c}38,729 \\
(8.2)\end{array}$ & $\begin{array}{c}38,729 \\
(8.2)\end{array}$ & $\begin{array}{c}180,162 \\
(6.8)\end{array}$ & $\begin{array}{c}180,162 \\
(6.8)\end{array}$ & $\begin{array}{c}33,772 \\
(18.1)\end{array}$ & $\begin{array}{c}33,772 \\
(18.1)\end{array}$ & $\begin{array}{l}42,494 \\
(18.8)\end{array}$ & $\begin{array}{c}42,494 \\
(18.8)\end{array}$ \\
\hline $91-95$ & $\begin{array}{c}22,229 \\
(4.7)\end{array}$ & $\begin{array}{c}22,229 \\
(4.7)\end{array}$ & $\begin{array}{c}90,050 \\
(3.4)\end{array}$ & $\begin{array}{c}90,050 \\
(3.4)\end{array}$ & $\begin{array}{c}27,166 \\
(14.6)\end{array}$ & $\begin{array}{c}27,166 \\
(14.6)\end{array}$ & $\begin{array}{c}34,779 \\
(15.4)\end{array}$ & $\begin{array}{c}34,779 \\
(15.4)\end{array}$ \\
\hline $96-99$ & $\begin{array}{c}6,007 \\
(1.3)\end{array}$ & $\begin{array}{c}6,007 \\
(1.3)\end{array}$ & $\begin{array}{c}21,804 \\
(0.8)\end{array}$ & $\begin{array}{c}21,804 \\
(0.8)\end{array}$ & $\begin{array}{c}9,728 \\
(5.2)\end{array}$ & $\begin{array}{c}9,728 \\
(5.2)\end{array}$ & $\begin{array}{c}11,615 \\
(5.1)\end{array}$ & $\begin{array}{c}11,615 \\
(5.1)\end{array}$ \\
\hline \multicolumn{9}{|l|}{ Sex } \\
\hline Female & $\begin{array}{c}255,638 \\
(54.3)\end{array}$ & $\begin{array}{c}255,638 \\
(54.3)\end{array}$ & $\begin{array}{c}1,494,273 \\
(56.0)\end{array}$ & $\begin{array}{c}1,494,271 \\
(56.0)\end{array}$ & $\begin{array}{c}119,501 \\
(64.0)\end{array}$ & $\begin{array}{c}119,501 \\
(64.0)\end{array}$ & $\begin{array}{c}148,245 \\
(65.6)\end{array}$ & $\begin{array}{c}148,245 \\
(65.6)\end{array}$ \\
\hline Male & $\begin{array}{c}214,984 \\
(45.7)\end{array}$ & $\begin{array}{c}214,984 \\
(45.7)\end{array}$ & $\begin{array}{c}1,173,658 \\
(44.0)\end{array}$ & $\begin{array}{c}1,173,652 \\
(44.0)\end{array}$ & $\begin{array}{c}67,170 \\
(36.0)\end{array}$ & $\begin{array}{c}67,170 \\
(36.0)\end{array}$ & $\begin{array}{c}77,725 \\
(34.4)\end{array}$ & $\begin{array}{c}77,725 \\
(34.4)\end{array}$ \\
\hline \multicolumn{9}{|l|}{ Race/Ethnicity } \\
\hline $\begin{array}{l}\text { American } \\
\text { Indian/Alaskan } \\
\text { Native }\end{array}$ & $\begin{array}{c}4,115 \\
(0.9)\end{array}$ & $\begin{array}{c}4,115 \\
(0.9)\end{array}$ & $\begin{array}{c}14,858 \\
(0.6)\end{array}$ & $\begin{array}{c}14,858 \\
(0.6)\end{array}$ & $\begin{array}{l}263 \\
(0.1)\end{array}$ & $\begin{array}{l}263 \\
(0.1)\end{array}$ & $\begin{array}{l}389 \\
(0.2)\end{array}$ & $\begin{array}{l}389 \\
(0.2)\end{array}$ \\
\hline
\end{tabular}




\begin{tabular}{|c|c|c|c|c|c|c|c|c|}
\hline $\begin{array}{l}\text { Asian/Pacific } \\
\text { Islander }\end{array}$ & $\begin{array}{c}11,587 \\
(2.5) \\
\end{array}$ & $\begin{array}{c}11,587 \\
(2.5)\end{array}$ & $\begin{array}{c}52,726 \\
(2.0)\end{array}$ & $\begin{array}{c}52,726 \\
(2.0)\end{array}$ & $\begin{array}{c}2,868 \\
(1.5) \\
\end{array}$ & $\begin{array}{c}2,868 \\
(1.5)\end{array}$ & $\begin{array}{c}2,339 \\
(1.0)\end{array}$ & $\begin{array}{c}2,339 \\
(1.0) \\
\end{array}$ \\
\hline Black & $\begin{array}{c}64,355 \\
(13.7)\end{array}$ & $\begin{array}{l}64,355 \\
(13.7)\end{array}$ & $\begin{array}{c}235,296 \\
(8.8)\end{array}$ & $\begin{array}{c}235,296 \\
(8.8)\end{array}$ & $\begin{array}{c}25,511 \\
(13.7)\end{array}$ & $\begin{array}{c}25,511 \\
(13.7)\end{array}$ & $\begin{array}{c}21,847 \\
(9.7)\end{array}$ & $\begin{array}{c}21,847 \\
(9.7) \\
\end{array}$ \\
\hline Hispanic & $\begin{array}{c}50,242 \\
(10.7)\end{array}$ & $\begin{array}{c}50,242 \\
(10.7)\end{array}$ & $\begin{array}{c}138,907 \\
(5.2)\end{array}$ & $\begin{array}{c}138,907 \\
(5.2)\end{array}$ & $\begin{array}{c}10,616 \\
(5.7)\end{array}$ & $\begin{array}{c}10,616 \\
(5.7)\end{array}$ & $\begin{array}{l}8,160 \\
(3.6)\end{array}$ & $\begin{array}{l}8,160 \\
(3.6)\end{array}$ \\
\hline $\begin{array}{l}\text { Non-Hispanic } \\
\text { White }\end{array}$ & $\begin{array}{c}329,199 \\
(69.9)\end{array}$ & $\begin{array}{c}329,199 \\
(69.9)\end{array}$ & $\begin{array}{c}2,156,397 \\
(80.8)\end{array}$ & $\begin{array}{c}2,156,389 \\
(80.8)\end{array}$ & $\begin{array}{c}146,674 \\
(78.6)\end{array}$ & $\begin{array}{c}146,674 \\
(78.6)\end{array}$ & $\begin{array}{c}192,426 \\
(85.2)\end{array}$ & $\begin{array}{c}192,426 \\
(85.2)\end{array}$ \\
\hline Other & $\begin{array}{c}3,627 \\
(0.8)\end{array}$ & $\begin{array}{c}3,627 \\
(0.8)\end{array}$ & $\begin{array}{c}18,005 \\
(0.7)\end{array}$ & $\begin{array}{c}18,005 \\
(0.7)\end{array}$ & $\begin{array}{l}452 \\
(0.2)\end{array}$ & $\begin{array}{c}452 \\
(0.2)\end{array}$ & $\begin{array}{c}468 \\
(0.2)\end{array}$ & $\begin{array}{c}468 \\
(0.2)\end{array}$ \\
\hline Unknown & $\begin{array}{l}7,497 \\
(1.6)\end{array}$ & $\begin{array}{l}7,497 \\
(1.6)\end{array}$ & $\begin{array}{c}51,742 \\
(1.9)\end{array}$ & $\begin{array}{c}51,742 \\
(1.9)\end{array}$ & $\begin{array}{l}287 \\
(0.2)\end{array}$ & $\begin{array}{l}287 \\
(0.2)\end{array}$ & $\begin{array}{l}341 \\
(0.2)\end{array}$ & $\begin{array}{l}341 \\
(0.2)\end{array}$ \\
\hline \multicolumn{9}{|l|}{$\begin{array}{l}\text { Low } \\
\text { Income/Disabled } \\
\text { Status }\end{array}$} \\
\hline $\begin{array}{l}\text { Low Income or } \\
\text { Disabled }\end{array}$ & $\begin{array}{c}188,443 \\
(40.0)\end{array}$ & $\begin{array}{c}188,443 \\
(40.0)\end{array}$ & $\begin{array}{c}839,214 \\
(31.5)\end{array}$ & $\begin{array}{c}839,214 \\
(31.5)\end{array}$ & $\begin{array}{c}123,380 \\
(66.1)\end{array}$ & $\begin{array}{c}123,380 \\
(66.1)\end{array}$ & $\begin{array}{c}123,488 \\
(54.6)\end{array}$ & $\begin{array}{c}123,488 \\
(54.6)\end{array}$ \\
\hline $\begin{array}{c}\text { Not Low } \\
\text { Income/Disabled }\end{array}$ & $\begin{array}{c}282,179 \\
(60.0)\end{array}$ & $\begin{array}{c}282,179 \\
(60.0)\end{array}$ & $\begin{array}{c}1,828,717 \\
(68.5)\end{array}$ & $\begin{array}{c}1,828,709 \\
(68.5)\end{array}$ & $\begin{array}{l}63,291 \\
(33.9)\end{array}$ & $\begin{array}{c}63,291 \\
(33.9)\end{array}$ & $\begin{array}{c}102,482 \\
(45.4)\end{array}$ & $\begin{array}{c}102,482 \\
(45.4)\end{array}$ \\
\hline \multicolumn{9}{|l|}{$\begin{array}{l}\text { Community vs } \\
\text { LTC/SNF }\end{array}$} \\
\hline Community & $\begin{array}{c}470,622 \\
(100.0)\end{array}$ & $\begin{array}{c}470,622 \\
(100.0)\end{array}$ & $\begin{array}{c}2,667,931 \\
(100.0)\end{array}$ & $\begin{array}{c}2,667,923 \\
(100.0)\end{array}$ & $\begin{array}{c}0 \\
(0.0)\end{array}$ & $\begin{array}{c}0 \\
(0.0)\end{array}$ & $\begin{array}{c}0 \\
(0.0)\end{array}$ & $\begin{array}{c}0 \\
(0.0)\end{array}$ \\
\hline $\begin{array}{l}\text { In Long term care } \\
\text { Facility or SNF }\end{array}$ & $\begin{array}{c}0 \\
(0.0)\end{array}$ & $\begin{array}{c}0 \\
(0.0)\end{array}$ & $\begin{array}{c}0 \\
(0.0)\end{array}$ & $\begin{array}{c}0 \\
(0.0)\end{array}$ & $\begin{array}{l}186,671 \\
(100.0)\end{array}$ & $\begin{array}{l}186,671 \\
(100.0)\end{array}$ & $\begin{array}{l}225,970 \\
(100.0)\end{array}$ & $\begin{array}{c}225,970 \\
(100.0)\end{array}$ \\
\hline \multicolumn{9}{|l|}{ RSI (9mo) } \\
\hline MEAN (SD) & $\begin{array}{l}0.048 \\
(.085)\end{array}$ & $\begin{array}{l}0.048 \\
(.085)\end{array}$ & $\begin{array}{l}0.038 \\
(.076)\end{array}$ & $\begin{array}{l}0.038 \\
(.076) \\
\end{array}$ & $\begin{array}{l}0.212 \\
(.136)\end{array}$ & $\begin{array}{l}0.212 \\
(.136)\end{array}$ & $\begin{array}{l}0.205 \\
(.140)\end{array}$ & $\begin{array}{l}0.205 \\
(.140)\end{array}$ \\
\hline \multicolumn{9}{|l|}{$\begin{array}{l}\text { Mortality } \\
\text { (Through Nov 30) }\end{array}$} \\
\hline Died & $\begin{array}{c}69,053 \\
(14.7)\end{array}$ & $\begin{array}{c}21,958 \\
(4.7)\end{array}$ & $\begin{array}{c}165,491 \\
(6.2)\end{array}$ & $\begin{array}{c}97,864 \\
(3.7)\end{array}$ & $\begin{array}{c}57,312 \\
(30.7)\end{array}$ & $\begin{array}{c}53,614 \\
(28.7)\end{array}$ & $\begin{array}{c}45,745 \\
(20.2)\end{array}$ & $\begin{array}{c}62,683 \\
(27.7)\end{array}$ \\
\hline Survived & $\begin{array}{c}401,569 \\
(85.3)\end{array}$ & $\begin{array}{c}448,664 \\
(95.3)\end{array}$ & $\begin{array}{c}2,502,440 \\
(93.8)\end{array}$ & $\begin{array}{c}2,570,059 \\
(96.3)\end{array}$ & $\begin{array}{c}129,359 \\
(69.3)\end{array}$ & $\begin{array}{c}133,057 \\
(71.3)\end{array}$ & $\begin{array}{c}180,225 \\
(79.8)\end{array}$ & $\begin{array}{c}163,287 \\
(72.3)\end{array}$ \\
\hline
\end{tabular}

309 Subjects were categorized as "LTC/SNF" if they received services in either a Long-Term Care (LTC) or Skilled Nursing

310 Facility (SNF) in February 2020, otherwise they were categorized as receiving services in the "Community." Confirmed 
311 Covid-19 cases were identified consistent with CMS guidance using ICD-10-CM codes for Covid-19 (B97.29 before April

3121,2020 and U07.1 thereafter) as a primary or secondary diagnosis between March 1, 2020 and September 30, 2020. ${ }^{12}$

313 Probable Covid-19 infection cases were identified using ICD-10-CM codes consistent with the CDC guidance (Z20.828)

314 and WHO recommendations (U07.2). ${ }^{13,14}$ The baseline risk of 9-month mortality defined by the Risk Stratification Index

315 (RSI) calculated on February 29, 2020. Beneficiaries receiving a diagnosis of probable or confirmed Covid-19 were

316 pairwise exactly matched 1:1 on Feb 29, 2020 with beneficiaries without a Covid-19 diagnosis based on sex, age (within

317 1-year), ethnicity, location of services in Feb 2020 (community or LTC/SNF), along with RSI as a propensity factor (within

$3180.1 \%$ ). The tabulated results demonstrate similarity of baseline characteristics between tightly matched populations. The

319 baseline risk of mortality (RSI) was much higher in patients categorized as LTC/SNF than Community subjects.

320

321 
medRxiv preprint doi: https://doi.org/10.1101/2021.04.07.21254793; this version posted April 10, 2021. The copyright holder for this preprint (which was not certified by peer review) is the author/funder, who has granted medRxiv a license to display the preprint in perpetuity.

\section{Excess Mortality Estimates}

323 The distribution of observed and expected mortality by diagnosis, category, and location

324 of care is presented in Fig 2. As expected, subjects with high baseline mortality risk in

325 the LTC/SNF cohort had actual mortality that exceeded all other groups. Those with

326 confirmed Covid-19 showed similarly increased mortality above expected levels in both

327 the LTC/SNF and community setting. Among community dwelling subjects, mortality

328 also exceeded expected risk in subjects with possible Covid-19.

329 Fig 2. Actual/Expected mortality plot for No Covid-19, Probable Covid-19, and 330 Confirmed Covid -19 cohorts in community, LTC/SNF, and combined analysis.

331 Panels A and B display actual and expected mortality (per 100,000 people) calculated

332 using different methods for Medicare subjects grouped by infection status and location

333 of services. Confirmed Covid-19 cases were identified consistent with CMS guidance

334 using ICD-10-CM codes for Covid-19 (B97.29 before April 1, 2020 and U07.1 thereafter)

335 as a primary or secondary diagnosis between March 1, 2020 and September 30, 336 2020. ${ }^{12}$ Probable Covid-19 infection cases were identified using ICD-10-CM codes

337 consistent with the CDC guidance (Z20.828) and WHO recommendations (U07.2). ${ }^{13,14}$

338 Subjects were categorized as "LTC/SNF" if they received services in either a Long Term

339 Care (LTC) or Skilled Nursing Facility (SNF) in February 2020, otherwise they were

340 categorized as receiving services in the "Community." Estimated mortality using RSI (A)

341 provides estimates consistent with actual mortality of historical controls (B).

343 There was an estimated excess of 130,702 (historical comparison method) or 101,482

344 (case matching method) deaths attributable to probable or confirmed Covid-19 across 
medRxiv preprint doi: https://doi.org/10.1101/2021.04.07.21254793; this version posted April 10, 2021. The copyright holder for this preprint (which was not certified by peer review) is the author/funder, who has granted medRxiv a license to display the preprint in perpetuity.

It is made available under a CC-BY-NC 4.0 International license.

345 the full population in the 9 months of 2020 that we considered. In the matched analysis,

346 half the deaths $(50,793)$ occurred in patients with a confirmed diagnosis of Covid-19 and

347 half $(50,689)$ occurred in those with a probable Covid-19 diagnosis. In contrast, 31,360

348 fewer subjects without a Covid-19 diagnosis died than expected, representing a $6 \%$

349 mortality

reduction

(Table

4). 
Table 4. Number of excess deaths by location, diagnosis, and method of calculation.

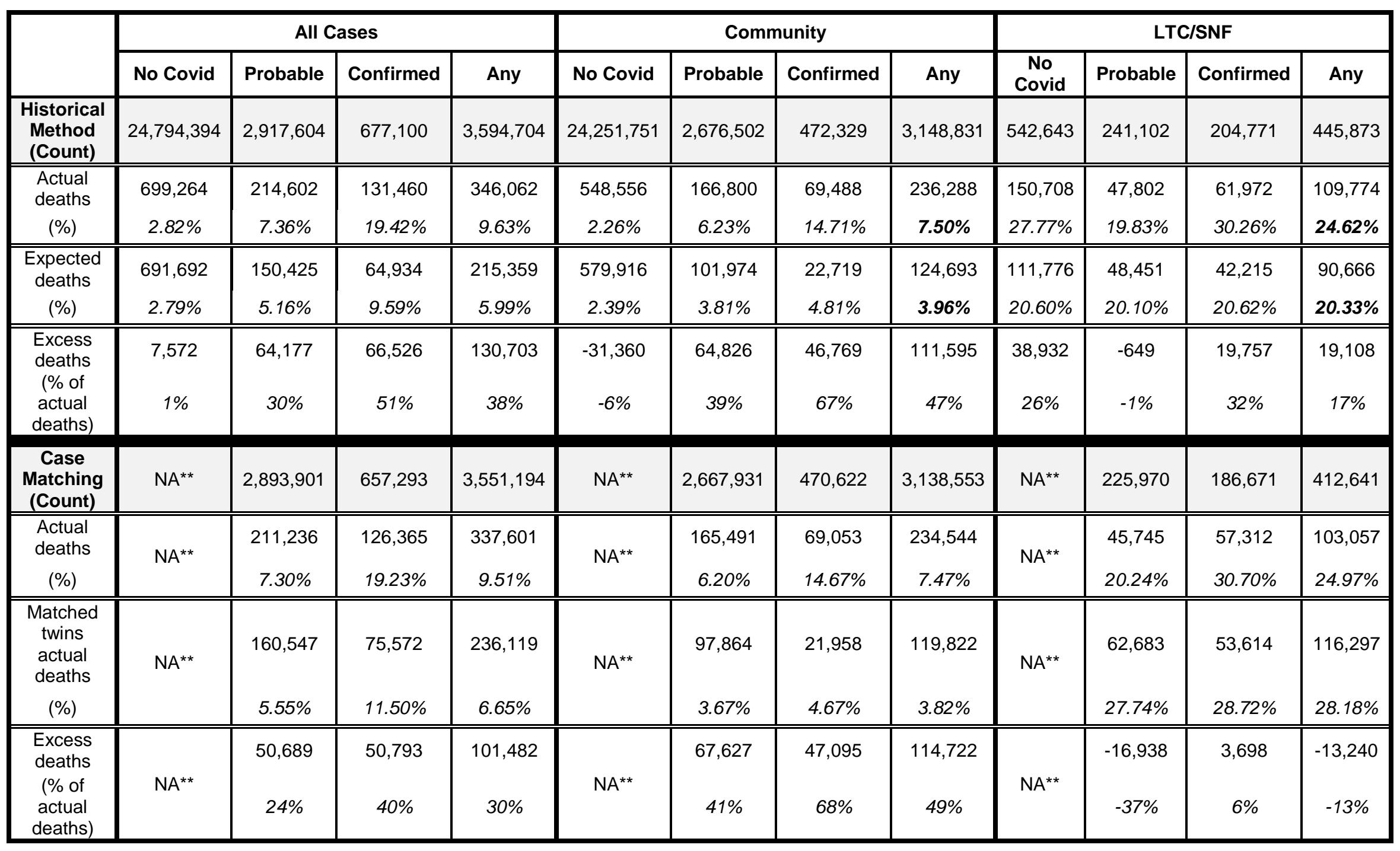


351 Covid-19 Confirmed and Probable groups are statistically significantly different. Historical Method: Expected deaths were

352 estimated as the sum of individual RSI-predicted 9-month mortality for the cohort. Case Matching Method: Expected

353 deaths were the actual deaths observed in the case-matched control populations..

354

$355{ }^{*}$ Excess deaths were calculated as the difference between actual deaths and expected deaths.

356 ** NA: No non-Covid controls were matched to non-Covid patients since the populations were the same.

357 Subjects were categorized as "LTC/SNF" if they received services in either a Long-Term Care (LTC) or Skilled Nursing 358 Facility (SNF) in February 2020, otherwise they were categorized as receiving services in the "Community." Confirmed 359 Covid-19 cases were identified consistent with CMS guidance using ICD-10-CM codes for Covid-19 (B97.29 before April 3601,2020 and U07.1 thereafter) as a primary or secondary diagnosis between March 1, 2020 and September 30, 2020. ${ }^{12}$ 361 Probable Covid-19 infection cases were identified using ICD-10-CM codes consistent with the CDC guidance (Z20.828) 362 and WHO recommendations (U07.2). ${ }^{13,14}$ The baseline risk of 9-month mortality defined by the Risk Stratification Index 363 (RSI) calculated on February 29, 2020. Two independent methods were used to estimate expected 2020 mortality as 364 described in the footnote above. The case matching (digital twin) method utilized the baseline risk of 9-month mortality 365 defined by the Risk Stratification Index (RSI). In this method, beneficiaries receiving a diagnosis of probable or confirmed 366 Covid-19 were pairwise exactly matched 1:1 on Feb 29, 2020 with beneficiaries without a Covid-19 diagnosis based on 367 sex, age (within 1-year), ethnicity, location of services in Feb 2020 (community or LTC/SNF), along with RSI as a 
propensity factor (within $0.1 \%$ ). The results demonstrate that, within the Medicare population, Covid- 19 had a devastating

369 impact by increasing mortality well above what would have been expected based on age and co-morbidities alone.

370677,100 (2.4\%) beneficiaries had confirmed Covid-19 and 2,917,604 (10.3\%) had probable Covid-19. 472,329 confirmed

371 cases were community living and 204,771 were in LTC. Mortality following a probable or confirmed diagnosis in the

372 community increased from an expected incidence of about $4 \%$ to actual incidence of $7.5 \%$. In long-term care facilities, the

373 corresponding increase was from $20.3 \%$ to $24.6 \%$. The absolute increase was therefore similar at $3-4 \%$ in the community

374 and in long-term care residents. But the percentage increase was far greater in the community (89\%) than among patients

375 in chronic care facilities $(21 \%)$ who had high baseline risk. The long-term care population without probable or confirmed

376 Covid-19 diagnoses experienced 38,932 excess deaths (35\%) compared to historical estimates. Limitations in access to

377 Covid-19 testing and disease under-reporting in long-term care patients probably contributed, although social isolation

378 and disruption in usual care presumably also contributed. Remarkably, there were 31,360 fewer deaths than expected in

379 community dwellers without probable or confirmed Covid-19 diagnoses, representing about a $6 \%$ reduction. Disruptions to

380 the healthcare system and avoided medical care were thus apparently offset by other factors, representing overall benefit.

381 The Covid-19 pandemic had marked effects on mortality, but the effects were highly context-dependent. 
medRxiv preprint doi: https://doi.org/10.1101/2021.04.07.21254793; this version posted April 10, 2021. The copyright holder for this preprint (which was not certified by peer review) is the author/funder, who has granted medRxiv a license to display the preprint in perpetuity.

\section{Public access}

383 Our model is highly predictive for mortality in Medicare beneficiaries with documented

384 Covid-19 infections. Because baseline RSI scores can help to identify Medicare

385 beneficiaries at highest risk for mortality due to Covid-19, we make the models publicly 386 available in the following formats:

1) Access to RSI risk calculators will be provided free of charge for authorized non-commercial uses via the HDAl API website (https://www.hdainstitute.com/api/).

2) Medicare beneficiaries or their health advocates may access their personalized health history and risk assessment by signing into Health Picture (https://my.healthhpicture.com). Health picture is an easy-to-use tool that allows Medicare beneficiaries and their family members a way to access their health histories and understand their Covid-19 risks.

3) Coefficients for a public version of a one-year RSI mortality model are provided at - (Risk Stratification Index | Cleveland Clinic).

\section{Discussion}

401 Age, sex, care location, and comorbidities were significant predictors of mortality. The 402 strongest individual predictor following a diagnosis of Covid-19 across all age 403 categories, and in both community and long-term care settings was the integrated 
medRxiv preprint doi: https://doi.org/10.1101/2021.04.07.21254793; this version posted April 10, 2021. The copyright holder for this preprint (which was not certified by peer review) is the author/funder, who has granted medRxiv a license to display the preprint in perpetuity. It is made available under a CC-BY-NC 4.0 International license.

404 measure of patient co-morbidities, RSI. Although many individual chronic conditions 405 were also significant risk factors in our unadjusted univariable analysis, the strength of 406 these associations were substantially diminished after adjustment for the primary 407 covariates of age, sex, race, location of services, and RSI. Our analysis is consistent 408 with previous work showing that older, non-white men, and patients receiving services 409 in long term care facilities are at special risk of dying from Covid-19 infections.

410 Using RSI as a composite measure of baseline mortality risk permitted precise case-

411 control matching, thereby allowing us to estimate excess deaths attributable Covid-19

412 by two complementary methods in Medicare recipients with probable or confirmed 413 Covid-19 diagnoses. Using the historical comparison, there was an increase from 414215,359 expected to 346,062 actual deaths, representing 130,702 excess deaths and a $41561 \%$ increase. Using matching, mortality increased from 236,119 expected to 337,601

416 observed deaths, representing 101,482 excess deaths and a $43 \%$ increase. Both 417 estimates far exceed the $15-20 \%$ excess mortality estimate reported in previous 418 analyses that included younger populations. Our results are therefore consistent with 419 the belief that older people are at much higher risk for developing severe Covid-19 420 illness - and of dying from it.

421 Overall, our historical model indicated that mortality following a probable or confirmed 422 diagnosis in the community increased from an expected incidence of about $4 \%$ to actual 423 incidence of $7.5 \%$. In LTC/SNF's, the corresponding increase was from $20.3 \%$ to $42424.6 \%$. Therefore, the absolute increase in mortality was similar at $3-4 \%$ in the 425 community and in long-term care residents. However, baseline risk (RSI) associated 426 with all individuals in a care setting varied greatly, being only about $2.6 \%$ in the 
medRxiv preprint doi: https://doi.org/10.1101/2021.04.07.21254793; this version posted April 10, 2021. The copyright holder for this preprint (which was not certified by peer review) is the author/funder, who has granted medRxiv a license to display the preprint in perpetuity. It is made available under a CC-BY-NC 4.0 International license .

427 community versus $20.5 \%$ in long-term care facilities. As a percentage, the relative

428 increase in mortality was thus far greater in the community (89\%) than among patients

429 in long term care facilities (21.0\%).

430 Somewhat remarkably, overall mortality decreased in Medicare participants without 431 probable or confirmed Covid-19 diagnoses. In fact, among community dwellers, there 432 were 31,360 fewer deaths than expected, representing about a $6 \%$ reduction.

433 Disruptions to the healthcare system and avoided medical care were thus apparently

434 offset by other factors, representing overall benefit. Obvious health benefits of pandemic

435 isolation include reduced exposure to other airborne illnesses such as influenza, fewer

436 driving accidents and fewer homicides. However, none seems sufficient to explain the

437 reduction. More subtle effects including reduced work or stress-related illness might

438 contribute more, although there is no obvious reason to believe that the pandemic

439 would reduce stress - especially in an over-65-year-old population.

440 The causes of reduced mortality in community dwelling Medicare participants remains

441 unclear. However, our results suggest that inadequate care for chronic conditions and

442 delayed care of acute events did not produce the feared outcome of higher short-term

443 mortality in the general population without Covid-19. But due to limited follow-up, we

444 caution that disruptions in healthcare delivery may yet result in adverse longer-term

445 outcomes due to delays in the diagnosis and treatment of new and existing chronic

446 conditions. An additional consideration is that prolonged sequela after severe Covid-19

447 infections (Long Covid syndrome) appear substantial and is an area requiring urgent 448 further study.(18) 
medRxiv preprint doi: https://doi.org/10.1101/2021.04.07.21254793; this version posted April 10, 2021. The copyright holder for this preprint (which was not certified by peer review) is the author/funder, who has granted medRxiv a license to display the preprint in perpetuity.

It is made available under a CC-BY-NC 4.0 International license .

449 There was a distinct disparity between community dwellers and those in long-term care

450 facilities with respect to historical mortality comparisons. In contrast to community

451 Medicare participants, the long-term care population without probable or confirmed

452 Covid-19 diagnoses experienced 38,932 excess deaths (35\%) compared to historical

453 estimates. We believe that limitations in access to Covid-19 testing and disease under-

454 reporting in long-term care patients probably were responsible for this finding. It seems

455 likely that many of the excess deaths in this vulnerable population were consequent to

456 undiagnosed Covid-19 infections. But it is also probable that social isolation and

457 disruption in usual care may have contributed as well. The higher-than-expected level of

458 excess deaths observed in this cohort (subjects without a probable or confirmed Covid

459 diagnosis) is reflected in our case matching results, which indicate a modest relative

460 reduction in deaths in subjects with a Covid related diagnosis. This is most likely due to

461 undiagnosed Covid cases included in the control population, but we cannot rule out the

462 possibility that the focus on care for the Covid patients had an unintended adverse

463 impact on the remaining population.

\section{Limitations}

466 We excluded less than $2.2 \%$ of the available population because of missing and

467 inconsistent values. Because data were missing non-systematically, exclusion of these

468 subjects was unlikely to introduce meaningful bias. We relied on administrative

469 diagnostic claims for Covid-19 to assign exposure. Surely these are inexact, especially

470 during our study period early in the pandemic. Furthermore, a new diagnostic code for 
medRxiv preprint doi: https://doi.org/10.1101/2021.04.07.21254793; this version posted April 10, 2021. The copyright holder for this preprint (which was not certified by peer review) is the author/funder, who has granted medRxiv a license to display the preprint in perpetuity.

It is made available under a CC-BY-NC 4.0 International license .

471 confirmed Covid-19 (U07.1) was introduced on April 1, 2020, and we assume that there

472 was some uncertainty regarding its proper application. However, Kadri et al recently

473 reported that this Covid-19 specific code showed high sensitivity and specificity

474 compared with the PCR test results.(19) A second limitation is that we did not account

475 for temporal changes in risk of exposure to Covid-19 in either setting, nor for

476 improvements in treatment of infected individuals over time. $(20,21)$

477 We assigned individuals to either community dwelling or long-term care subgroups

478 based on coding in February 2020. Some participants undoubtedly changed their care

479 settings during the analysis period. Skilled nursing facilities, for example, include

480 patients who remain semi-permanently along with others who stay for short periods

481 such during rehabilitation from major orthopedic procedures before resuming

482 community life. But among patients who died, $79 \%$ of those who were in a long-term

483 care facility on the anchor date of Feb 29, 2020 had long term care charges within two

484 months of death.

485 Our analysis was based on $28,389,098$ adults enrolled in the US fee-for-service and

486 Medicare/Medicaid program. The results are therefore broadly applicable to Medicare

487 eligible adults. Although our sample included a fair number of dual eligible subjects

488 below age 65 , our results should only be cautiously generalized to younger and 489 healthier populations.

\section{Summary}

491 Mortality following a probable or confirmed Covid-19 diagnosis in the community 492 increased from an expected incidence of about $4 \%$ to actual incidence of $7.5 \%$. In long- 
medRxiv preprint doi: https://doi.org/10.1101/2021.04.07.21254793; this version posted April 10, 2021. The copyright holder for this preprint (which was not certified by peer review) is the author/funder, who has granted medRxiv a license to display the preprint in perpetuity. It is made available under a CC-BY-NC 4.0 International license .

493 term care facilities, the corresponding increase was from $20.3 \%$ to $24.6 \%$. The absolute

494 increase was therefore similar at $3-4 \%$ in the community and in long-term care

495 residents. But the percentage increase was far greater in the community (89\%) than

496 among patients in chronic care facilities (21\%) who had high baseline risk.

497 The long-term care population without probable or confirmed Covid-19 diagnoses

498 experienced 38,932 excess deaths (35\%) compared to historical estimates. Limitations

499 in access to Covid-19 testing and disease under-reporting in long-term care patients

500 probably contributed, although social isolation and disruption in usual care presumably

501 contributed. Remarkably, there were 31,360 fewer deaths than expected in community

502 dwellers without probable or confirmed Covid-19 diagnoses, representing about a $6 \%$

503 reduction. Disruptions to the healthcare system and avoided medical care were thus

504 apparently offset by other factors, representing overall benefit.

505 The Covid-19 pandemic had marked effects on mortality, but the effects were highly

506 context-dependent. Among community dwelling Medicare participants with suspected or

507 confirmed Covid-19 diagnoses, mortality nearly doubled, but from a relatively low

508 baseline. Patients in long-term care facilities had a similar absolute increase in mortality,

509 but because their baseline mortality was $20.5 \%$, the relative increase was smaller. In

510 contrast, community dwelling Medicare participants without COVID had about $6 \%$ lower-

511 than-expected mortality. 


\section{Acknowledgements}

515 Author Contributions: Greenwald and Chamoun had full access to all the data in the

516 study and take responsibility for the integrity of the data and the accuracy of the data

517 analysis.

518 Data Curation: Chamoun, Greenwald

519 Formal Analysis: Greenwald, Chamoun

520 Funding Acquisition: Chamoun, Gray

521 Investigation: Chamoun, Greenwald

522 Methodology: Manberg, Sessler, Chamoun

523 Project Administration: Greenwald, Clain

524 Resources: Chamoun

525 Software: Chamoun, Greenwald

526 Supervision: Manberg, Greenwald, Chamoun

527 Validation: Sessler, Maheshwari, Manberg, Gray, Clain

528 Visualization: Clain, Greenwald

529 Writing - Original Draft Preparation: Manberg, Greenwald, Sessler 
medRxiv preprint doi: https://doi.org/10.1101/2021.04.07.21254793; this version posted April 10, 2021. The copyright holder for this preprint (which was not certified by peer review) is the author/funder, who has granted medRxiv a license to display the preprint in perpetuity. It is made available under a CC-BY-NC 4.0 International license .

530 Writing - Review and Editing: Sessler, Maheshwari, Gray, Clain, Manberg, Chamoun,

531 Greenwald

532 Conflict of Interest Disclosures: Greenwald, Chamoun, Manberg, Clain and Gray are

533 employees of and hold equity interest in Health Data Analytics Institute. Dr. Sessler is a 534 paid consultant of and holds equity interest in Health Data Analytics Institute. Dr.

535 Maheshwari received no compensation from Health Data Analytics Institute.

536 Funding/Support: The study was funded by Health Data Analytics Institute. The Robert

537 Wood Johnson Foundation provides support for providing access to the Covid-19 RSI 365

538 risk prediction models by academic researchers for non-commercial purposes via the

539 HDAI API (https://www.hda-institute.com/api/).

540 Role of Funder/Sponsor: Other than the authors and Data Analyst Douceur Tengu, no

541 additional members of Health Data Analytics had a role in the design and conduct of the

542 study; collection, management, analysis, and interpretation of the data; preparation,

543 review, or approval of the manuscript; or decision to submit the manuscript for

544 publication.

545

546 We thank John Parks, Zhenyu Hong and Douceur Tengu (Data Analysts, Health Data

547 Analytics Institute) for their assistance in developing models and preparing figures and

548 tables. 


\section{References}

550 1. CDC. CDC COVID Data Tracker: Centers for Disease Control and Prevention;

$5512021 \quad$ [Available from: https://covid.cdc.gov/covid-data-

552 tracker/\#cases_casesper100klast7days.

553 2. Chen YH, Glymour MM, Catalano R, Fernandez A, Nguyen T, Kushel M, et al.

554 Excess Mortality in California During the Coronavirus Disease 2019 Pandemic, March to

555 August 2020. JAMA Intern Med. 2020.

556 3. Faust JS, Krumholz HM, Du C, Mayes KD, Lin Z, Gilman C, et al. All-Cause

557 Excess Mortality and COVID-19-Related Mortality Among US Adults Aged 25-44 Years,

558 March-July 2020. JAMA. 2020.

559 4. Lauren M. Rossen AMB, Farida B. Ahmad, Paul Sutton, Robert N. Anderson.

560 Excess Deaths Associated with COVID-19, by Age and Race and Ethnicity - United

561 States, January 26-October 3, 20202021 [Available from:

562 https://www.cdc.gov/mmwr/volumes/69/wr/mm6942e2.htm.

563 5. CDC. Medical Conditions and Risk of for Severe COVID-19 IIIness: Centers for

564 Disease Control and Prevention; 2021 [Available from:

565 https://www.cdc.gov/coronavirus/2019-ncov/need-extra-precautions/people-with-

566 medical-conditions.html.

567 6. Wang Q, Davis PB, Gurney ME, Xu R. COVID-19 and dementia: Analyses of 568 risk, disparity, and outcomes from electronic health records in the US. Alzheimers

569 Dement. 2021. 
medRxiv preprint doi: https://doi.org/10.1101/2021.04.07.21254793; this version posted April 10, 2021. The copyright holder for this preprint (which was not certified by peer review) is the author/funder, who has granted medRxiv a license to display the preprint in perpetuity. It is made available under a CC-BY-NC 4.0 International license .

$5707 . \quad$ Harrison SL, Fazio-Eynullayeva E, Lane DA, Underhill P, Lip GYH. Comorbidities

571 associated with mortality in 31,461 adults with COVID-19 in the United States: A

572 federated electronic medical record analysis. PLoS Med. 2020;17(9):e1003321.

573 8. Wang Q, Berger NA, Xu R. Analyses of Risk, Racial Disparity, and Outcomes

574 Among US Patients With Cancer and COVID-19 Infection. JAMA Oncol. 2021;7(2):220-

5757.

576 9. Wynants L, Van Calster B, Collins GS, Riley RD, Heinze G, Schuit E, et al.

577 Prediction models for diagnosis and prognosis of covid-19 infection: systematic review

578 and critical appraisal. BMJ. 2020;369:m1328.

579 10. Experton B, Tetteh HA, Lurie N, Walker P, Carroll CJ, Elena A, et al. A Predictive

580 Model for Severe Covid-19 in the Medicare Population: A Tool for Prioritizing Scarce

581 Vaccine Supply. medRxiv. 2020:2020.10.28.20219816.

582 11. Dun C, Walsh CM, Bae S, Adalja A, Toner E, Lash TA, et al. A Machine Learning 583 Study of 534,023 Medicare Beneficiaries with COVID-19: Implications for Personalized

584 Risk Prediction. medRxiv. 2020:2020.10.27.20220970.

585 12. von Elm E, Altman DG, Egger M, Pocock SJ, Gotzsche PC, Vandenbroucke JP, 586 et al. The Strengthening the Reporting of Observational Studies in Epidemiology 587 (STROBE) statement: guidelines for reporting observational studies. Lancet. $588 \quad 2007 ; 370(9596): 1453-7$.

589 13. CMS. Preliminary Medicare COVID-19 Data Snapshot | CMS: Centers for

590 Medicare \& Medicaid Services; 2021 [Available from: https://www.cms.gov/research-

591 statistics-data-systems/preliminary-medicare-covid-19-data-snapshot. 
medRxiv preprint doi: https://doi.org/10.1101/2021.04.07.21254793; this version posted April 10, 2021. The copyright holder for this preprint (which was not certified by peer review) is the author/funder, who has granted medRxiv a license to display the preprint in perpetuity. It is made available under a CC-BY-NC 4.0 International license .

592 14. CDC. ICD-10-CM Official Coding and Reporting Guidelines April 1, 2020 through

593 September 30, 2020. Centers for Disease Control and Prevention; 2020.

594 15. WHO. COVID-19 coding in ICD-10: World Health Organization; 2020 [cited World

595 Health Organization. Available from: https://www.who.int/classifications/icd/COVID-19-

596 coding-icd10.pdf.

597 16. Chamoun GF, Li L, Chamoun NG, Saini V, Sessler DI. Validation and Calibration 598 of the Risk Stratification Index. Anesthesiology. 2017;126(4):623-30.

599 17. CMS. Condition Categories - Chronic Conditions Data Warehouse: Centers for 600 Medicare \& Medicaid Services; 2021 [Available from:

601 https://www2.ccwdata.org/web/guest/condition-categories.

602 18. Lopez-Leon S, Wegman-Ostrosky T, Perelman C, Sepulveda R, Rebolledo PA, 603 Cuapio A, et al. More than 50 Long-term effects of Covid-19: a systematic review and 604 meta-analysis. medRxiv preprint. 2021.

605 19. Kadri SS, Gundrum J, Warner S, Cao Z, Babiker A, Klompas M, et al. Uptake 606 and Accuracy of the Diagnosis Code for COVID-19 Among US Hospitalizations. JAMA. 607 2020;324(24):2553-4.

608 20. Nguyen NT, Chinn J, Nahmias J, Yuen S, Kirby KA, Hohmann S, et al. Outcomes 609 and Mortality Among Adults Hospitalized With COVID-19 at US Medical Centers. JAMA

610 Netw Open. 2021;4(3):e210417.

611 21. Tenforde MW, Fisher KA, Patel MM. Identifying COVID-19 Risk Through 612 Observational Studies to Inform Control Measures. JAMA. 2021. 


\section{Supporting Information}

616 S1 Fig. Consort style waterfall flowchart detailing population selection

617 methodology. Confirmed Covid-19 cases were identified consistent with CMS

618 guidance using ICD-10-CM codes for Covid-19 (B97.29 before April 1, 2020 and U07.1

619 thereafter) as a primary or secondary diagnosis between March 1, 2020 and September

$62030,2020 .{ }^{12}$ Probable Covid-19 infection cases were identified using ICD-10-CM codes

621 consistent with the CDC guidance (Z20.828) and WHO recommendations (U07.2). ${ }^{13,14}$

622 Subjects were excluded for missing data if values for any baseline characteristic used in

623 the study were missing (i.e., age, sex, ethnicity, location of care, zip code derived

624 measures, dates of coverage, or baseline risk of 9 month mortality assessed with the

625 Risk Stratification Index (RSI).) Additionally, we excluded subjects whose records had 626 inconsistent values among source files containing similar variables such as birth date 627 and sex.

628

629 S2 Fig. Performance characteristics of RSI model. Panel A: ROC curve, Panel B 630 Calibration plot, Panel C Sensitivity and Positive Predicted Value vs probability of

631 mortality. (A) Area Under the Receiver Operating Curve (AuROC) for the development

632 Learn Set (80\% of 2018 Set) was 0.88 (95\% Confidence Interval of [0.88-0.88]). AuROC

633 for the prospective Test Set (20\% of 2018 Set) was 0.88 (95\% Confidence Interval of

$634[0.88-0.88]$. Similar performance in the Test Set compared to the Learn Set supports a 635 lack of overfitting in the development of the predictor. 
medRxiv preprint doi: https://doi.org/10.1101/2021.04.07.21254793; this version posted April 10, 2021. The copyright holder for this preprint (which was not certified by peer review) is the author/funder, who has granted medRxiv a license to display the preprint in perpetuity. It is made available under a CC-BY-NC 4.0 International license .

636 (B) The calibration plot displays the mean actual vs predicted 1 year mortality for 637 populations clustered in increments of $1 \%$ probability of mortality. Dark green, light 638 green, and red dots are populations of the lowest $95 \%, 95 \%-99 \%$, and top $1 \%$ risk of 639 mortality. The diagonal line identifies the domain of ideal performance where actual and 640 expected mortality rates are equal for a population. The performance of this index is 641 very close to ideal performance for approximately $99 \%$ of the population. Tabulated 642 metrics: The sample size in this test set $(\mathrm{N})$ was $11,923,144$ with an incidence of $1 \mathrm{yr}$ 643 mortality (Event_Test) of $4.5 \%$. The Slope and Intercept (INT) fit of the data are 0.94 644 and 0.01 , respectively. The area under the Receiver Operating Curve was 0.88 . The 645 Mean Average Error (MEA) from cluster coordinates (i.e., (expected, actual) couplets) to 646 the identity line was calculated for the database divided into populations grouped from 647 the riskiest to least risky subjects using cluster sizes ranging from 1 (i.e., each individual 648 as a cluster) to 1000 neighboring subjects (e.g., MAE to MAE_1000). The $95 \%$

649 Confidence Interval $(\mathrm{Cl})$ for the fits of these populations to the identify line is tabulated 650 (i.e., AE_Cl to AE_Cl_1000). Rsq_unit is a goodness of fit measure of individual results 651 to the ideal line.

652 (C) Positive Predictive Accuracy (blue dots) and Sensitivity (purple dots) versus the 653 fraction of population, sorted by the risk of 1 year mortality. The vertical red line 654 indicates where the number of patients above the risk threshold equals the incidence of 655 mortality in the population. There are several metrics tabulated in the figure: The area 656 under the Receiver Operating Characteristic $(\mathrm{ROC})$ is 0.88 . The incidence of mortality 657 (IR) in the population of $11,891,922$ was $4.4 \%$. Vertical bars help identify the PPA and 658 sensitivity performance for detectors operating to identify the riskiest $5 \%, 10 \%$, and $20 \%$ 
medRxiv preprint doi: https://doi.org/10.1101/2021.04.07.21254793; this version posted April 10, 2021. The copyright holder for this preprint (which was not certified by peer review) is the author/funder, who has granted medRxiv a license to display the preprint in perpetuity. It is made available under a CC-BY-NC 4.0 International license .

659 patients. The PPV, sensitivity and relative risk (RR) are tabulated for these detector 660 operating points.

664 B) on 2020 population. ROC curves for all subjects, No Covid-19, Probable Covid-

66519 and Confirmed Covid-19 populations. Confirmed Covid-19 cases were identified 666 consistent with CMS guidance using ICD-10-CM codes for Covid-19 (B97.29 before 667 April 1, 2020 and U07.1 thereafter) as a primary or secondary diagnosis between March 6681,2020 and September 30, 2020. ${ }^{12}$ Probable Covid-19 infection cases were identified 669 using ICD-10-CM codes consistent with the CDC guidance (Z20.828) and WHO 670 recommendations (U07.2) ${ }^{13,14}$ (A,B) ROCs display the sensitivity vs. 1 - specificity in

671 detecting patients who died within 9 months after prediction from February 29,2020 672 (baseline). The areas under each ROC, with their corresponding 95\% confidence

673 intervals, are tabulated in the lower right of each figure. Predictions using RSI yielded

674 better performance $(\mathrm{A})$ than those using a model based on age, sex and chronic 675 conditions (B).

678 S4 Fig. Forest plot showing the relative risk and $95 \% \mathrm{Cl}$ of significant predictors 679 of confirmed Covid-19 infection. Confirmed Covid-19 cases were identified consistent 680 with CMS guidance using ICD-10-CM codes for Covid-19 (B97.29 before April 1, 2020 681 and U07.1 thereafter) as a primary or secondary diagnosis between March 1, 2020 and 
medRxiv preprint doi: https://doi.org/10.1101/2021.04.07.21254793; this version posted April 10, 2021. The copyright holder for this preprint (which was not certified by peer review) is the author/funder, who has granted medRxiv a license to display the preprint in perpetuity. It is made available under a CC-BY-NC 4.0 International license .

682 September 30, 2020. ${ }^{12}$ Subjects were categorized as "LTC/SNF" if they received 683 services in either a Long Term Care (LTC) or Skilled Nursing Facility (SNF) in February

684 2020, otherwise they were categorized as receiving services in the "Community."

685 Predictors were assessed at baseline (February 29, 2020) and include quintiles of Risk

686 Stratification Index (RSI), presence of chronic conditions, location of services (LTC/SNF

687 vs Community), and demographic variables (i.e., age, sex, race, and quintiles of median

688 household income imputed by zip code according to 2015 Census data.) Variables not

689 remaining in the adjusted model are indicated by the presence of empty parenthesis

690 under the adjusted odds ratio. Location of services, age, status of end-stage renal

691 disease (ESRD) and RSI were the strongest (unadjusted) predictors of infection.

692 Location of services and ESRD remained strong predictors following adjustment;

693 however, risks associated with having chronic conditions were typically reduced when

694 adjusted by the presence of other factors.

695

696

697 S5 Fig. Observed mortality rates by age and RSI quintiles. Rates of mortality within

6989 months following baseline (February 29, 2020) in Medicare subpopulations

699 categorized by age, location of services, infection status, and quintiles of the baseline

700 risk of mortality assessed using the Risk Stratification Index (RSI). Confirmed Covid-19

701 cases were identified consistent with CMS guidance using ICD-10-CM codes for Covid-

70219 (B97.29 before April 1, 2020 and U07.1 thereafter) as a primary or secondary

703 diagnosis between March 1, 2020 and September 30, 2020. ${ }^{12}$ Probable Covid-19

704 infection cases were identified using ICD-10-CM codes consistent with the CDC 
705 guidance (Z20.828) and WHO recommendations (U07.2). ${ }^{13,14}$ Subjects were

706 categorized as "LTC/SNF" if they received services in either a Long-Term Care (LTC) or

707 Skilled Nursing Facility (SNF) in February 2020, otherwise they were categorized as

708 receiving services in the "Community." As expected, subjects in quintiles with higher

709 baseline risk of mortality had higher rates of observed mortality. For subjects without a

710 Covid diagnosis, mortality rates were lower in the community setting compared to those

711 in the LTC/SNF; however, for subjects with confirmed or probable Covid infection,

712 mortality rates were typically higher in the community setting than in the LTC/SNF.

713

714 S1 File. RSI Development Method for 9-month predictions.

715 
RSI9 (mo) Highest $20 \%$ vs Lowest $20 \% 17.0(16.3,17.8)$ Age: $96-99$ vs $66-70$

$6.55(6.32,6.80)$

RSI9 (mo) $60-80 \%$ vs Lowest $20 \%$

$5.78(5.53,6.05)$

Age: $91-95$ vs $66-70$

$5.14(5.01,5.28)$

Age: $86-90$ vs $66-70$

$4.06(3.96,4.16)$

RSI9(mo) $40-60 \%$ vs Lowest $20 \%$

$3.29(3.13,3.46)$

$3.00(2.93,3.08)$

$\begin{array}{lr}\text { Services Setting: L TC/SNF vs Communit } 2.52(2.48,2.55) \\ \text { Dementia } & 2.23(2.20,2.26)\end{array}$

Age: $76-80$ vs $66-70$

$2.10(2.05,2.15)$

Lung Cancer

Acute Myocardiac Infarction

$2.06(1.97,2.16)$

$2.05(1.97,2.14)$

$1.92(1.89,1.96)$

$1.89(1.80,2.00)$

$1.83(1.81,1.85)$

$1.82(1.75,1.89)$

$1.81(1.79,1.84)$

$1.79(1.77,1.82)$

$1.79(1.76,1.82)$

$1.72(1.67,1.76)$

End-Stage Renal Disease

$\begin{array}{lr}\text { Chronic Obstructive Pulmonary Disease } 1.66(1.64,1.69) \\ \text { Stroke or ThA } & 1.65(1.62 .1 .69)\end{array}$

Ischemic Heart Disease

$1.53(1.51,1.55)$

Benign Prostatic Hyperplasia

$1.53(1.50,1.55)$

$1.51(1.44,1.58)$

$1.46(1.44 .1 .48)$

$1.44(1.42,1.46)$

$1.37(1.34,1.41)$

$1.34(1.30 .1 .38)$

$1.33(1.29,1.37)$

$1.29(1.27,1.30)$

$1.26(1.24 .1 .27)$

$1.24(1.16,1.33)$

$1.22(1.20,1.23)$

$1.20(1.18,1.22)$

$1.19(1.15,1.23)$

$1.16(1.15 .1 .18)$

$1.14(1.05,1.25)$

$1.10(1.08,1.11)$

$1.09(1.06,1.11)$

$1.04(1.03 .1 .05)$

$1.03(0.96,1.10)$

$1.02(1.00,1.05)$

$0.98(0.96,1.00)$

$0.94(0.92,0.96)$

$0.93(0.91,0.95)$

$0.92(0.90,0.94)$

$0.91(0.89,0.93)$

$0.91(0.88,0.94)$

$0.91(0.90,0.92)$

$0.86(0.85,0.88)$

$0.84(0.82,0.86)$

$0.66(0.64,0.69)$

$0.43(0.40,0.46)$
Age: $81-85$ vs $66-70$
H $8.38(8.01,8.78)$

3.25 (3.12, 3.38)

$4.04(3.85,4.23)$

$2.50(2.43,2.58)$

$2.03(1.98,2.09)$

$2.61(2.49,2.75)$

$1.72(1.68,1.77)$

$1.08(1.06,1.10)$

$1.16(1.13,1.18)$

$1.50(1.46,1.54)$

$1.48(1.41,1.56)$

$1.22(1.16,1.27)$

( . )

$1.66(1.58,1.75)$

$1.05(1.04,1.07)$

$($,

$1.06(1.05,1.08)$

$1.16(1.14,1.18)$

$1.06(1.04,1.08)$

$1.30(1.26,1.34)$

$1.10(1.09 .1 .12)$

$1.02(1.00,1.04)$

$($,

$1.03(1.01,1.05)$

$1.05(1.01,1.11)$

$1.07(1.06,1.09)$

$0.98(0.96,0.99)$

$1.27(1.24,1.30)$

$0.85(0.82,0.88)$

$($,

$1.06(1.04,1.07)$

$1.44(1.42 .1 .46)$

$1.66(1.55,1.79)$

$0.94(0.93,0.96)$

$1.31(1.28,1.34)$

$1.31(1.25,1.36)$

(, )

$1.16(1.06,1.27)$

$0.98(0.96,0.99)$

$($, )

$0.92(0.90,0.93)$

$1.18(1.10,1.27)$

$1.28(1.25,1.31)$

$0.95(0.93,0.97)$

$0.97(0.95,0.99)$

$0.97(0.95,0.99)$

$0.97(0.96,0.99)$

$0.94(0.93,0.96)$

(, )

$1.15(1.13,1.17)$

$0.91(0.89,0.92)$

$0.95(0.93,0.98)$

$0.58(0.55,0.60)$

$0.94(0.87,1.01)$
$95 \% \mathrm{Cl})$

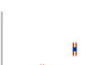

H

H

H

1

H

H

,

d

1

H

H

H

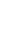

$\begin{array}{ll}\text { Age: } 18-55 \text { vs } 66-70 & 0.66(0.64,0.69) \\ \text { Race: Unknown vs White } & 0.43(0.40,0.46)\end{array}$


(A)

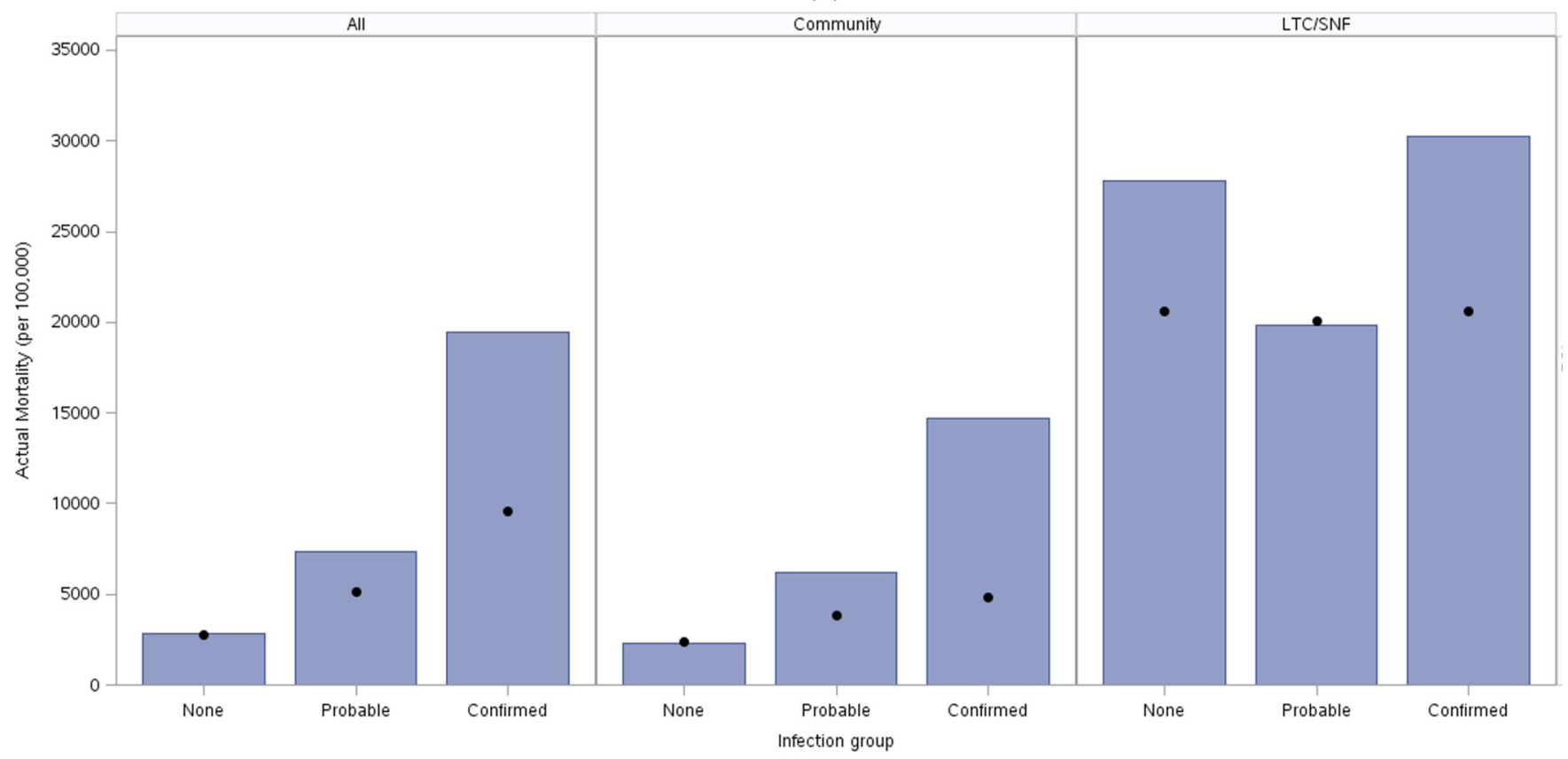

(B)

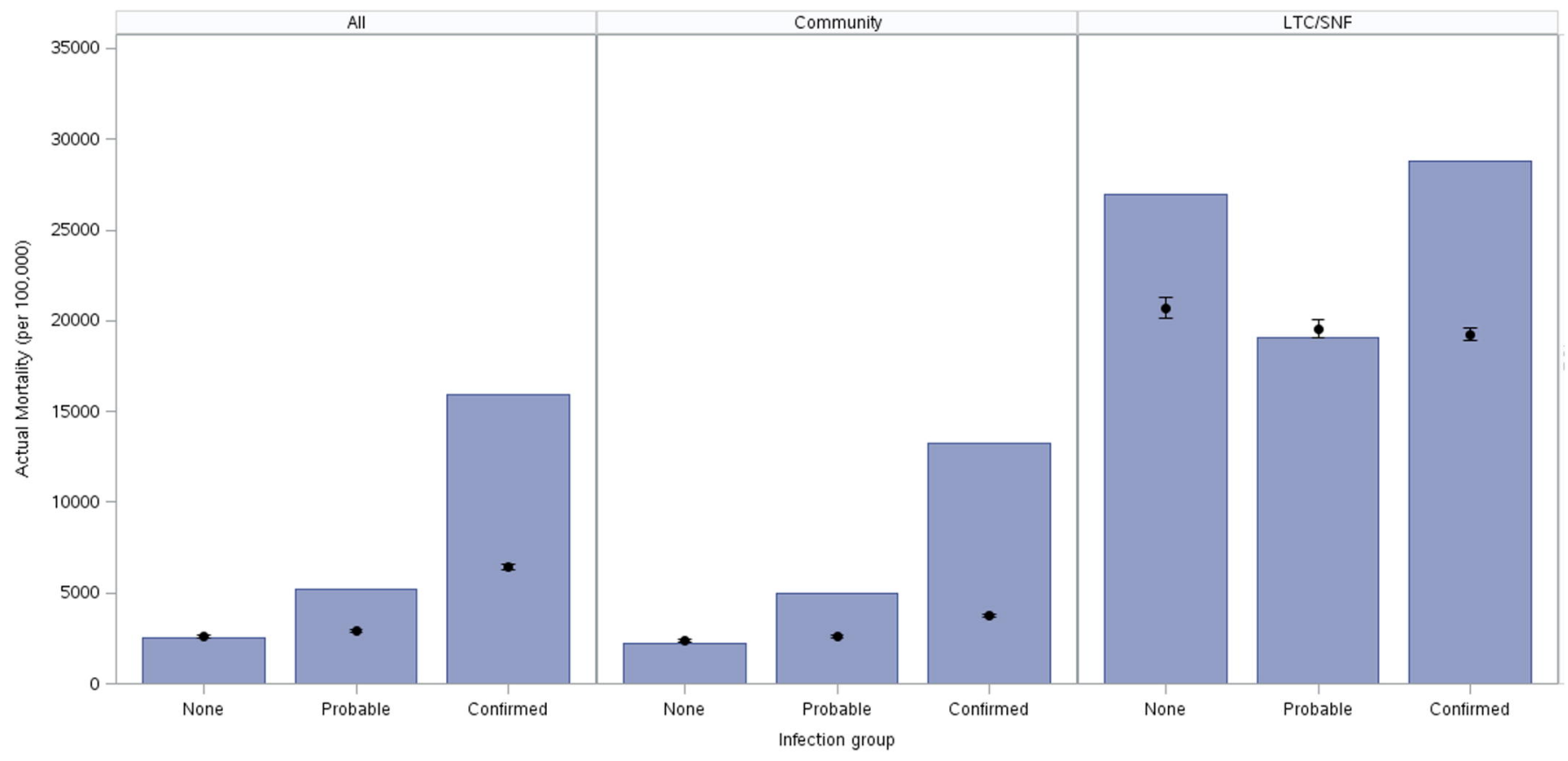

